\title{
Normed Interval Space and Its Topological Structure
}

\author{
Hsien-Chung Wu \\ Department of Mathematics, National Kaohsiung Normal University, Kaohsiung 802, Taiwan; \\ hcwu@nknucc.nknu.edu.tw
}

Received: 6 July 2019; Accepted: 11 October 2019; Published: 16 October 2019

\begin{abstract}
Based on the natural vector addition and scalar multiplication, the set of all bounded and closed intervals in $\mathbb{R}$ cannot form a vector space. This is mainly because the zero element does not exist. In this paper, we endow a norm to the interval space in which the axioms are almost the same as the axioms of conventional norm by involving the concept of null set. Under this consideration, we shall propose two different concepts of open balls. Based on the open balls, we shall also propose the different types of open sets, which can generate many different topologies.
\end{abstract}

Keywords: interval space; open sets; norms; null set; open balls

MSC: 46A19; 15A03

\section{Introduction}

In the real world, collecting data becomes an important issue for solving the practical and large-scaled problems in engineering, economics, and social sciences. However, in most situations, the data cannot be collected precisely. For example, the level of water cannot be measured precisely owing to the fluctuation. In this case, we may assume that the level of water is in a bounded closed interval that can represent the uncertainty. Also, in the financial market, stock price fluctuates violently, such that it may not be reasonable to record the price as an exact real number in a short period time. In this case, we can also assume that the stock price is located in a bounded closed interval within a short period of time. In other words, the bounded closed intervals can be used to describe the uncertain data instead of using probability theory. In this case, the bounded closed intervals can be regarded as the so-called interval numbers, which can be treated as "points", not as closed intervals. Therefore, the arithmetic and related properties of the interval numbers should be established. For detailed discussion we refer the to the monographs [1-5].

The interval analysis may provide a useful tool to tackle uncertainty when the problems in engineering, economics and social sciences are formulated as interval-valued problems. For example, the interval-valued optimization problems and the interval-valued solution concepts of cooperative games have been studied in [6-9] and the references therein. The interval-valued problems can also be abstractly formulated as nonlinear problems in infinitely dimensional space. Therefore, the techniques of functional analysis and nonlinear analysis should be used to study those interval-valued problems. In this case, the basic concepts like topological vector space and normed space should be established on the interval space. In this paper, we are going to generate the topologies of normed interval space, although the interval space cannot form a vector space.

The set of all bounded closed intervals in $\mathbb{R}$ cannot form a vector space. This is primarily because each bounded closed interval does not have an additive inverse element, which will be presented more clearly in the context of this paper. The topic of functional analysis is based on the vector space. We may try to develop the theory of functional analysis based on the interval space. Therefore, the first pioneering work is to create the Hahn-Banach extension theorem based on the interval space 
by referring to $\mathrm{Wu}[10]$, as the conventional Hahn-Banach extension theorem in functional analysis is very useful in nonlinear analysis, vector optimization and mathematical economics. In this paper, even though the interval space $\mathcal{I}$ cannot be a vector space, we still can endow a norm to the set of all bounded closed intervals by involving the concept of null set, and to study its topological structure.

As any real number $x \in \mathbb{R}$ can be regarded as a bounded closed interval $[x, x], \mathbb{R}$ is contained in $\mathcal{I}$. Given $A=\left[a^{L}, a^{U}\right]$ and $B=\left[b^{L}, b^{U}\right]$ in $\mathcal{I}$, the interval addition is given by

$$
A \oplus B=\left[a^{L}+b^{L}, a^{U}+b^{U}\right]
$$

and the scalar multiplication in $\mathcal{I}$ is given by

$$
k A= \begin{cases}{\left[k a^{L}, k a^{U}\right]} & \text { if } k \geq 0 \\ {\left[k a^{U}, k a^{L}\right]} & \text { if } k<0 .\end{cases}
$$

By the above definition, we have

$$
-A=-\left[a^{L}, a^{U}\right]=\left[-a^{U},-a^{L}\right] .
$$

For any $\lambda \in \mathbb{R}$ and $A, B \in \mathcal{I}$,

$$
\lambda(A \oplus B)=\lambda A \oplus \lambda B .
$$

Let $A$ and $B$ be two bounded closed intervals. The subtraction between $A$ and $B$ is denoted and defined by

Now, we have

$$
A \ominus B \equiv A \oplus(-B)=\left[a^{L}-b^{U}, a^{U}-b^{L}\right] .
$$

$$
A \ominus A=\left[a^{L}, a^{U}\right] \ominus\left[a^{L}, a^{U}\right]=\left[a^{L}, a^{U}\right] \oplus\left[-a^{U},-a^{L}\right]=\left[a^{L}-a^{U}, a^{U}-a^{L}\right],
$$

which says that the additive inverse element in $\mathcal{I}$ does not exist. This is partly because the concept of "zero element" of $\mathcal{I}$ is not defined. This also states that $\mathcal{I}$ cannot form a vector space under the above interval addition and scalar multiplication. Based on this inspiration, the following set,

$$
\Omega=\{A \ominus A: A \in \mathcal{I}\}
$$

is called the null set of $\mathcal{I}$, which can be regarded as a kind of "zero element" of $\mathcal{I}$. We also see that the true zero element of $\mathcal{I}$ is $[0,0]$, as $A \oplus[0,0]=A$ for any $A \in \mathcal{I}$.

In this paper, we shall endow a norm to $\mathcal{I}$, in which the axioms are almost the same as the axioms of conventional norm. The only difference is that the concept of null set is involved in the axioms. To study the topological structure of $(\mathcal{I},\|\cdot\|)$, we need to consider the open balls. Let us recall that if $(X,\|\cdot\|)$ is a (conventional) normed space, then we see that

$$
\{y:\|x-y\|<\epsilon\}=\{x+z:\|z\|<\epsilon\}
$$

by taking $y=x+z$. However, for spaces $(\mathcal{I},\|\cdot\|)$ and $A, B, C \in \mathcal{I}$,

$$
\{B:\|A \ominus B\|<\epsilon\} \neq\{A \oplus C:\|C\|<\epsilon\}
$$

in general. This is because by taking $B=A \oplus C$, we have

$$
\|A \ominus B\|=\|A \ominus(A \oplus C)\|=\|\omega \ominus C\| \neq\|C\|,
$$


where $\omega=A \ominus A \in \Omega$. In this case, two types of open balls will be considered in $(\mathcal{I},\|\cdot\|)$. Therefore, many types of open sets will also be considered. Based on the different types of openness, we shall study the topological structure of the normed interval space $(\mathcal{I},\|\cdot\|)$.

Now, we recall the Embedding Theorem obtained by Rådström [11] as follows. Let $M$ be a commutative semigroup such that the law of cancellation holds true in $M$, i.e., the following conditions are satisfied; for $a, b, c \in M$,

- $(a+b)+c=a+(b+c)$

- $a+b=b+a$;

- $a+c=b+c$ implies $a=b$.

We also assume that the non-negative scalar multiplication in $M$ satisfies the following conditions.

- $\lambda(a \oplus b)=\lambda a \oplus \lambda b$ for $a, b \in M$ and $\lambda \geq 0$;

- $\quad\left(\lambda_{1}+\lambda_{2}\right) a=\lambda_{1} a \oplus \lambda_{2} a$ for $a \in M$ and $\lambda_{1}, \lambda_{2} \geq 0$;

- $\quad \lambda_{1}\left(\lambda_{2} a\right)=\left(\lambda_{1} \lambda_{2}\right) a$ for $a \in M$ and $\lambda_{1}, \lambda_{2} \geq 0$;

- $1 a=a$ for $a \in M$.

Then we define the set $N$ consisting of the equivalence classes of pairs $(a, b)$ of elements of $M$. The equivalence relation is defined by

$$
(a, b) \sim(c, d) \text { if and only if } a+d=b+c .
$$

The equivalence class containing the pair $(a, b)$ is denoted by $\langle a, b\rangle$. It is clear to see that if $(c, d) \in\langle a, b\rangle$, then $\langle a, b\rangle=\langle c, d\rangle$, i.e., $a+d=b+c$. The addition in $N$ is defined by

$$
\langle a, b\rangle+\langle c, d\rangle=\langle a+c, b+d\rangle,
$$

and the scalar multiplication in $N$ is defined by

$$
\lambda\langle a, b\rangle= \begin{cases}\langle\lambda a, \lambda b\rangle & \text { if } \lambda \geq 0 \\ \langle|\lambda| b,|\lambda| a\rangle=\langle-\lambda b,-\lambda a\rangle & \text { if } \lambda<0 .\end{cases}
$$

Then, $N$ is a vector space.

Suppose that $M$ is endowed with a metric $\delta$ satisfying the following conditions,

- $\delta(a \oplus c, b \oplus c)=\delta(a, b)$ for $a, b, c \in M$, i.e., $\delta$ is invariant under translation;

- $\delta(\lambda a, \lambda b)=\lambda \delta(a, b)$ for $\lambda \geq 0$ and $a, b \in M$, i.e., $\delta$ is positively homogeneous,

We also define a metric $\hat{\delta}$ in $N$ by

$$
\hat{\delta}(\langle a, b\rangle,\langle c, d\rangle)=\delta(a \oplus d, b \oplus c) .
$$

Then, $(N, \hat{\delta})$ forms a metric space. Let $\Theta$ be the zero element of $N$. We define

$$
\|\langle a, b\rangle\|=\hat{\delta}(\langle a, b\rangle, \Theta) .
$$

Then, $\|\langle a, b\rangle\|=\delta(a, b)$ and $(N,\|\cdot\|)$ forms a normed space.

For any fixed $b_{0} \in M$, we define the function $\pi: M \rightarrow N$ by $\pi(a)=\left\langle a \oplus b_{0}, b_{0}\right\rangle$. Then, we have the following properties.

- $\pi$ is one-to-one.

- $\pi(a \oplus b)=\pi(a)+\pi(b)$ for $a, b \in M$.

- $\pi(\lambda a)=\lambda \pi(a)$ for $\lambda \geq 0$ and $a \in M$. 
Moreover, $M$ can be embedded into the normed space $(N,\|\cdot\|)$ isomorphically and isometrically via the embedding function $\pi$ such that $M$ spans $N$.

The embedding theorem presented by Rådström [11] says that the family $\mathcal{I}$ consisting of all bounded closed intervals can be be embedded into a normed space isomorphically and isometrically. The differences between the approach by Rådström [11] and the approach in this paper are given below.

- According the approach by Rådström [11], the topological structure can be established in the normed space $N$. As the embedding function $\pi$ is isometric, we can recover the metric in $M$ to establish the topological structure. However, this topological structure may be complicated to be expressed analytically via the embedding function $\pi$. In this paper, the topological structures in $\mathcal{I}$ can be analytically expressed.

- As the embedding approach of Rådström [11] is based on a specific normed space, $N$, defined above, the topological structures established in $N$ is specific. Therefore, the topological structures in $M$ recovered from the embedding function $\pi$ is also specific. However, the topological structures in $\mathcal{I}$ can be freely established in this paper based on the different kinds of informal norms. In other words, we can have many topological structures corresponding to the different types of informal norms.

- As we mentioned above, the embedding approach by Rådström [11] is based on a specific normed space $N$, which says that $M$ can be endowed with a norm-like (as $M$ is a not a vector space) via the embedding function $\pi$. In this case, the normed-like space $M$ has a specific form restricted by the specific normed space $N$. However, in this paper, we can freely define any so-called informal norms in $\mathcal{I}$ using the null sets.

In Section 2, many useful properties of interval spaces are presented in order to study the topology generated by the norm. In Section 3, we introduce the concept of norms involving the concept of null set and provide many useful properties for further investigation. In Section 4, we provide the nonintuitive properties for the open balls. In Section 5, we propose many types of open sets based on the different types of open balls. Finally, in Section 6, we investigate the topologies generated by these different types of open sets.

\section{Interval Spaces}

Let us recall that the following set,

$$
\Omega=\{A \ominus A: A \in \mathcal{I}\},
$$

is called the null set of $\mathcal{I}$, which can be regarded as a kind of "zero element" of $\mathcal{I}$. For further discussion, we present some useful properties.

Remark 1. We have the following observations.

- It is clear that

$$
\Omega=\{[-k, k]: k \geq 0\}=\{k[-1,1]: k \geq 0\},
$$

as

$$
A \ominus A=\left[a^{L}, a^{U}\right] \ominus\left[a^{L}, a^{U}\right]=\left[a^{L}, a^{U}\right] \oplus\left[-a^{U},-a^{L}\right]=\left[a^{L}-a^{U}, a^{U}-a^{L}\right],
$$

where $a^{U}-a^{L} \geq 0$.

- $\omega \in \Omega$ implies $-\omega=\omega$.

- $A s[x, x] \in \mathcal{I},[x, x] \ominus[x, x]=[0,0] \in \Omega$.

- $\lambda \Omega=\Omega$ for $\lambda \in \mathbb{R}$ with $\lambda \neq 0$.

- $\Omega$ is closed under the interval addition; that is, $\omega_{1} \oplus \omega_{2} \in \Omega$ for any $\omega_{1}, \omega_{2} \in \Omega$. 
As the null set $\Omega$ can be regarded as a kind of "zero element", we can propose the almost identical concept for elements in $\mathcal{I}$.

Definition 1. Given any $A, B \in \mathcal{I}$, we say that $A$ and $B$ are "almost identical" if and only if there exist $\omega_{1}, \omega_{2} \in \Omega$ such that $A \oplus \omega_{1}=B \oplus \omega_{2}$. In this case, we write $A \stackrel{\Omega}{=} B$.

For $A \ominus B=C$, we cannot have $A=B \oplus C$. However, we can have $A \stackrel{\Omega}{=} B \oplus C$. Indeed, as $A \ominus B=C$, by adding $B$ on both sides, we obtain $A \oplus \omega=B \oplus C$, where $\omega=B \ominus B \in \Omega$. Therefore, $A \stackrel{\Omega}{=} B \oplus C$.

Proposition 1. The binary relation $\stackrel{\varrho}{=}$ is an equivalence relation.

Proof. The reflexivity and symmetry are obvious. We just claim the transitivity. Suppose that $A \stackrel{\Omega}{=} B$ and $B \stackrel{\Omega}{=}$. There exist $\omega_{1}, \omega_{2}, \omega_{3}, \omega_{4} \in \Omega$, such that $A \oplus \omega_{1}=B \oplus \omega_{2}$ and $B \oplus \omega_{3}=C \oplus \omega_{4}$. Therefore, we have

$$
A \oplus \omega_{1} \oplus \omega_{3}=B \oplus \omega_{2} \oplus \omega_{3}=B \oplus \omega_{3} \oplus \omega_{2}=C \oplus \omega_{2} \oplus \omega_{4} .
$$

As $\Omega$ is closed under the addition, it follows that $A \stackrel{\Omega}{=}$ and the proof is complete.

As $\stackrel{\Omega}{=}$ is an equivalence relation, we can obtain the quotient space $\mathcal{I} / \stackrel{\Omega}{=}$, where $[A] \in \mathcal{I} / \stackrel{\Omega}{=}$ means

$$
[A]=\{B \in \mathcal{I}: A \stackrel{\Omega}{=} B\} .
$$

It is clear to see that $[\omega]=\Omega$ for any $\omega \in \Omega$. Now, we define

$$
[A]+[B]=[A \oplus B] \text { and } k[A]=[k A] \text { for any } k \in \mathbb{R} .
$$

Then, we see that $\Omega$ is a zero element of the quotient space $\mathcal{I} / \stackrel{\Omega}{=}$ satisfying $[A]+\Omega=[A]$. However, still, the quotient space $\mathcal{I} / \stackrel{\Omega}{=}$ cannot be a vector space. Given any $\alpha, \beta \in \mathbb{R}$ with $\alpha \cdot \beta<0$ and $A \in \mathcal{I}$, we see that

$$
(\alpha+\beta) A \neq \alpha A \oplus \beta A
$$

in general. Therefore, in general, we also have

$$
(\alpha+\beta)[A]=[(\alpha+\beta) A] \neq[\alpha A \oplus \beta A]=\alpha[A]+\beta[A],
$$

which says that $\mathcal{I} / \stackrel{\Omega}{=}$ cannot be a vector space.

Proposition 2. The following statements hold true.

(i) If $A \ominus B \in \Omega$, then $A \stackrel{\Omega}{=} B$.

(ii) If $A \stackrel{\Omega}{=} B$, then there exists $\omega \in \Omega$, such that $A \ominus B \oplus \omega \in \Omega$.

Proof. To prove part (i), we have $A \oplus(-B)=\omega_{1}$ for some $\omega_{1} \in \Omega$, which implies $A \oplus(-B) \oplus B=$ $\omega_{1} \oplus B$ by adding $B$ on both sides. This shows that $A \oplus \omega_{2}=\omega_{1} \oplus B$ for $\omega_{1}, \omega_{2} \in \Omega$.

To prove part (ii), as $A \stackrel{\Omega}{=} B$, we have $A \oplus \omega_{2}=\omega_{1} \oplus B$ for some $\omega_{1}, \omega_{2} \in \Omega$. By adding $-B$ on both sides, we obtain $A \ominus B \oplus \omega_{2}=\omega_{1} \oplus \omega_{3} \in \Omega$, where $\omega_{3}=B \ominus B \in \Omega$. This completes the proof.

Proposition 3. The following statements hold true. 
(i) Given any nondegenerated interval, $A \in \mathcal{I}$, there exists $\bar{A} \in \mathcal{I}$ and $\omega \in \Omega$ with $\omega \neq[0,0]$, such that $A=\bar{A} \oplus \omega$.

(ii) We have $\Omega \oplus \Omega=\Omega$. Given any subset $\mathcal{A}$ of $\mathcal{I}$, let $\overline{\mathcal{A}}=\mathcal{A} \oplus \Omega$. Then, $\overline{\mathcal{A}} \oplus \Omega=\overline{\mathcal{A}}$.

(iii) Given any $A, \bar{A} \in \mathcal{I}$, we have $A \oplus \omega=\bar{A} \oplus \omega$ that implies $A=\bar{A}$ for any $\omega \in \Omega$. Given any subset $\mathcal{A}$ of $\mathcal{I}$, we have $A \oplus \omega \in \mathcal{A} \oplus \omega$ that implies $A \in \mathcal{A}$ for any $\omega \in \Omega$.

(iv) Given any $\omega_{1}, \omega_{2} \in \Omega$, we have $\omega_{1}=\omega \oplus \omega_{2}$ or $\omega_{2}=\omega \oplus \omega_{1}$ for some $\omega \in \Omega$.

(v) Given any $\omega \in \Omega$ with $\omega \neq[0,0]$, we have $\omega=\omega_{1} \oplus \omega_{2}$ for some $\omega_{1}, \omega_{2} \in \Omega$ with $\omega_{1} \neq[0,0]$ and $\omega_{2} \neq[0,0]$.

(vi) Given any $\omega=[-k, k] \in \Omega$ and any $\omega_{1}=\left[-k_{1}, k_{1}\right] \in \Omega$ with $k_{1}<k$, we have $\omega=\omega_{1} \oplus \omega_{2}$ for $\omega_{2}=\left[k_{1}-k, k-k_{1}\right] \in \Omega$.

Proof. To prove part (i), for nondegenerated interval $A=\left[a^{L}, a^{U}\right]$, we can take $0<k<\left(a^{U}-a^{L}\right) / 2$ and $\bar{A}=\left[a^{L}+k, a^{U}-k\right]$. In this case, we have

$$
A=\left[a^{L}, a^{U}\right]=\left[a^{L}+k, a^{U}-k\right] \oplus[-k, k]=\bar{A} \oplus \omega,
$$

where $\omega=[-k, k] \in \Omega$.

To prove part (ii), it is obvious that $\Omega \oplus \Omega \subseteq \Omega$. Now, for any $\omega=[-k, k] \in \Omega$ for $k>0$, we can write $k=k_{1}+k_{2}$ for some $k_{1}, k_{2}>0$. Then

$$
\omega=[-k, k]=\left[-\left(k_{1}+k_{2}\right), k_{1}+k_{2}\right]=\left[-k_{1}, k_{1}\right] \oplus\left[-k_{2}, k_{2}\right],
$$

where $\omega_{1}=\left[-k_{1}, k_{1}\right], \omega_{2}=\left[-k_{2}, k_{2}\right] \in \Omega$. This says that $\Omega \subseteq \Omega \oplus \Omega$. Therefore we obtain $\Omega \oplus \Omega=\Omega$. On the other hand, we have

$$
\overline{\mathcal{A}} \oplus \Omega=\mathcal{A} \oplus \Omega \oplus \Omega=\mathcal{A} \oplus \Omega=\overline{\mathcal{A}}
$$

Parts (iii)-(vi) are obvious. This completes the proof.

The following interesting results will be used for discussing the topological structure of normed interval space.

Proposition 4. Let $\mathcal{A}_{1}$ and $\mathcal{A}_{2}$ be subsets of $\mathcal{I}$. Then the following inclusion is satisfied:

$$
\left(\mathcal{A}_{1} \cap \mathcal{A}_{2}\right) \oplus \Omega \subseteq\left[\left(\mathcal{A}_{1} \oplus \Omega\right) \cap\left(\mathcal{A}_{2} \oplus \Omega\right)\right] .
$$

If we further assume that $\mathcal{A}_{1} \oplus \Omega \subseteq \mathcal{A}_{1}$ and $\mathcal{A}_{2} \oplus \Omega \subseteq \mathcal{A}_{2}$, then the following equality is satisfied:

$$
\left[\left(\mathcal{A}_{1} \oplus \Omega\right) \cap\left(\mathcal{A}_{2} \oplus \Omega\right)\right]=\left(\mathcal{A}_{1} \cap \mathcal{A}_{2}\right) \oplus \Omega .
$$

Proof. For $B \in\left(\mathcal{A}_{1} \cap \mathcal{A}_{2}\right) \oplus \Omega$, we have $B=A \oplus \omega$ with $A \in \mathcal{A}_{i}$ for $i=1,2$ and $\omega \in \Omega$, which also says that $B \in\left[\left(\mathcal{A}_{1} \oplus \Omega\right) \cap\left(\mathcal{A}_{2} \oplus \Omega\right)\right]$, i.e., $\left(\mathcal{A}_{1} \cap \mathcal{A}_{2}\right) \oplus \Omega \subseteq\left[\left(\mathcal{A}_{1} \oplus \Omega\right) \cap\left(\mathcal{A}_{2} \oplus \Omega\right)\right]$.

Under the further assumption, let $A \in\left(\mathcal{A}_{1} \oplus \Omega\right) \cap\left(\mathcal{A}_{2} \oplus \Omega\right)$. Then

$$
A=A_{1} \oplus \omega_{1}=A_{2} \oplus \omega_{2}
$$

for some $A_{1} \in \mathcal{A}_{1}, A_{2} \in \mathcal{A}_{2}$ and $\omega_{1}, \omega_{2} \in \Omega$. By part (iv) of Proposition 3, we have $\omega_{1}=\omega \oplus \omega_{2}$ or $\omega_{2}=\omega \oplus \omega_{1}$ for some $\omega \in \Omega$. Now, we consider the following cases.

- $\quad$ Assume that $\omega_{2}=\omega \oplus \omega_{1}$. Let $\widehat{A}_{2}=A_{2} \oplus \omega$. Then $\widehat{A}_{2} \in \mathcal{A}_{2} \oplus \Omega \subseteq \mathcal{A}_{2}$, i.e., $\widehat{A}_{2} \in \mathcal{A}_{2}$. In this case, we obtain

$$
\widehat{A}_{2} \oplus \omega_{1}=A_{2} \oplus \omega \oplus \omega_{1}=A_{2} \oplus \omega_{2}=A_{1} \oplus \omega_{1}=A \in \mathcal{A}_{1} \oplus \omega_{1}
$$


which implies $\widehat{A}_{2} \in \mathcal{A}_{1}$ by part (iii) of Proposition 3. This says that $\widehat{A}_{2} \in \mathcal{A}_{1} \cap \mathcal{A}_{2}$, i.e., $A=$ $\widehat{A}_{2} \oplus \omega_{1} \in\left(\mathcal{A}_{1} \cap \mathcal{A}_{2}\right) \oplus \Omega$.

- Assume that $\omega_{1}=\omega \oplus \omega_{2}$. Let $\widehat{A}_{1}=A_{1} \oplus \omega$. Then $\widehat{A}_{1} \in \mathcal{A}_{1} \oplus \Omega \subseteq \mathcal{A}_{1}$, i.e, $\widehat{A}_{1} \in \mathcal{A}_{1}$. In this case, we obtain

$$
\widehat{A}_{1} \oplus \omega_{2}=A_{1} \oplus \omega \oplus \omega_{2}=A_{1} \oplus \omega_{1}=A_{2} \oplus \omega_{2}=A \in \mathcal{A}_{2} \oplus \omega_{2},
$$

which implies $\widehat{A}_{1} \in \mathcal{A}_{2}$ by part (iii) of Proposition 3. This says that $\widehat{A}_{1} \in \mathcal{A}_{1} \cap \mathcal{A}_{2}$, i.e., $A=$ $\widehat{A_{1}} \oplus \omega_{2} \in\left(\mathcal{A}_{1} \cap \mathcal{A}_{2}\right) \oplus \Omega$.

This completes the proof.

\section{Normed Interval Spaces}

Many kinds of norms on $\mathcal{I}$ are proposed below, which will involve the concept of null set.

Definition 2. Given the non-negative real-valued function $\|\cdot\|: \mathcal{I} \rightarrow \mathbb{R}_{+}$, we consider the following conditions.

(i) $\|\alpha A\|=|\alpha|\|A\|$ for any $A \in \mathcal{I}$ and $\alpha \in \mathbb{F}$;

(i') $\|\alpha A\|=|\alpha|\|A\|$ for any $A \in \mathcal{I}$ and $\alpha \in \mathbb{F}$ with $\alpha \neq 0$.

(ii) $\|A \oplus B\| \leq\|A\|+\|B\|$ for any $A, B \in \mathcal{I}$.

(iii) $\|A\|=0$ implies $A \in \Omega$.

We say that $\|\cdot\|$ satisfies the null condition when condition (iii) is replaced by $\|A\|=0$ if and only if $A \in \Omega$. Different kinds of normed interval spaces are defined below.

- We say that $(\mathcal{I},\|\cdot\|)$ is a pseudo-seminormed interval space if and only if conditions ( $\left.i^{\prime}\right)$ and (ii) are satisfied.

- We say that $(\mathcal{I},\|\cdot\|)$ is a seminormed interval space if and only if conditions (i) and (ii) are satisfied.

- We say that $(\mathcal{I},\|\cdot\|)$ is a pseudo-normed interval space if and only if conditions ( $\left.i^{\prime}\right)$, (ii), and (iii) are satisfied.

- We say that $(\mathcal{I},\|\cdot\|)$ is a normed interval space if and only if conditions (i)-(iii) are satisfied.

Now we consider the following conditions:

- We say that $\|\cdot\|$ satisfies the null superinequality if and only if $\|A \oplus \omega\| \geq\|A\|$ for any $A \in \mathcal{I}$ and $\omega \in \Omega$.

- We say that $\|\cdot\|$ satisfies the null sub-inequality if and only if $\|A \oplus \omega\| \leq\|A\|$ for any $A \in \mathcal{I}$ and $\omega \in \Omega$.

- We say that $\|\cdot\|$ satisfies the null equality if and only if $\|A \oplus \omega\|=\|A\|$ for any $A \in \mathcal{I}$ and $\omega \in \Omega$.

Example 1. Let us define a non-negative real-valued function $\|\cdot\|$ on $\mathcal{I}$ by

$$
\left\|\left[a^{L}, a^{U}\right]\right\|=\left|a^{L}+a^{U}\right| .
$$

Then $(\mathcal{I},\|\cdot\|)$ is a normed interval space in which the null condition is satisfied.

Example 2. Let us define a non-negative real-valued function $\|\cdot\|$ on $\mathcal{I}$ by

$$
\left\|\left[a^{L}, a^{U}\right]\right\|=a^{U}-a^{L} .
$$

Then $(\mathcal{I},\|\cdot\|)$ is a seminormed interval space in which the null condition is not satisfied.

Example 3. Let us define a non-negative real-valued function $\|\cdot\|$ on $\mathcal{I}$ by

$$
\left\|\left[a^{L}, a^{U}\right]\right\|=\max \left\{\left|a^{L}+a^{U}\right|, a^{U}-a^{L}\right\} .
$$


Then $(\mathcal{I},\|\cdot\|)$ is a seminormed interval space. We only prove that the triangle inequality is satisfied. Given $A=\left[a^{L}, a^{U}\right]$ and $B=\left[b^{L}, b^{U}\right]$, we have

$$
\begin{aligned}
\|A \oplus B\| & =\left\|\left[a^{L}+b^{L}, a^{U}+b^{U}\right]\right\|=\max \left\{\left|a^{L}+b^{L}+a^{U}+b^{U}\right|, a^{U}+b^{U}-a^{L}-b^{L}\right\} \\
& \leq \max \left\{\left|a^{L}+b^{L}\right|+\left|a^{U}+b^{U}\right|,\left(a^{U}-a^{L}\right)+\left(b^{U}-b^{L}\right)\right\} \\
& \leq \max \left\{\left|a^{L}+a^{U}\right|, a^{U}-a^{L}\right\}+\max \left\{\left|b^{L}+b^{U}\right|, b^{U}-b^{L}\right\}=\|A\|+\|B\| .
\end{aligned}
$$

On the other hand, since $A \ominus B=-(B \ominus A)$ for any $A, B \in \mathcal{I}$, it is clear to see that

$$
\|A \ominus B\|=\|B \ominus A\|
$$

Proposition 5. Let $(\mathcal{I},\|\cdot\|)$ be a pseudo-seminormed interval space such that $\|\cdot\|$ satisfies the null superinequality. For any $A, C, B_{1}, \cdots, B_{m} \in \mathcal{I}$, we have

$$
\|A \ominus C\| \leq\left\|A \ominus B_{1}\right\|+\left\|B_{1} \ominus B_{2}\right\|+\cdots+\left\|B_{j} \ominus B_{j+1}\right\|+\cdots+\left\|B_{m} \ominus C\right\| .
$$

Proof. We have

$$
\|A \ominus C\| \leq\left\|A \oplus(-C) \oplus B_{1} \oplus \cdots \oplus B_{m} \oplus\left(-B_{1}\right) \oplus \cdots \oplus\left(-B_{m}\right)\right\|
$$

(using the null superinequality for $m$ times)

$$
\begin{aligned}
& =\left\|\left(A \oplus\left(-B_{1}\right)\right) \oplus\left(B_{1} \oplus\left(-B_{2}\right)\right)+\cdots+\left(B_{j} \oplus\left(-B_{j+1}\right)\right)+\cdots+\left(B_{m} \oplus(-C)\right)\right\| \\
& \leq\left\|A \ominus B_{1}\right\|+\left\|B_{1} \ominus B_{2}\right\|+\cdots+\left\|B_{j} \ominus B_{j+1}\right\|+\cdots+\left\|B_{m} \ominus C\right\|
\end{aligned}
$$

(by the triangle inequality).

This completes the proof.

\section{Open Balls}

If $(X,\|\cdot\|)$ is a (conventional) normed space, then we see that

$$
\{y:\|x-y\|<\epsilon\}=\{x+z:\|z\|<\epsilon\}
$$

by taking $y=x+z$. Let $(\mathcal{I},\|\cdot\|)$ be a seminormed interval space. Then, the following equality,

$$
\{B:\|A \ominus B\|<\epsilon\}=\{A \oplus C:\|C\|<\epsilon\}
$$

does not hold. The reason is that, by taking $B=A \oplus C$ and using (1), we can only have

$$
\|A \ominus B\|=\|A \ominus(A \oplus C)\|=\|-C \oplus \omega\| \neq\|C\|,
$$

where $\omega=A \ominus A \in \Omega$. Therefore, we shall define two types of open balls, as follows.

Definition 3. Let $(\mathcal{I},\|\cdot\|)$ be a pseudo-seminormed interval space. Two types of open balls with radius $\epsilon$ are defined by

$$
\mathcal{B}^{\diamond}(A ; \epsilon)=\{A \oplus C:\|C\|<\epsilon\}
$$

and

$$
\mathcal{B}(A ; \epsilon)=\{B:\|A \ominus B\|=\|B \ominus A\|<\epsilon\} .
$$

Remark 2. Let $(\mathcal{I},\|\cdot\|)$ be a pseudo-seminormed interval space. Then, we have some interesting observations. 
- For any $A \in \mathcal{I}$, the equality $\|A \ominus A\|=0$ does not necessarily hold true, unless $\|\cdot\|$ satisfies the null condition. In other words, if $\|\cdot\|$ satisfies the null condition, then $A \in \mathcal{B}(A ; \epsilon)$. In Example 2, if we define $\left\|\left[a^{L}, a^{U}\right]\right\|=a^{U}-a^{L}$, then the null conditions is not satisfied, which says that $A \notin \mathcal{B}(A ; \epsilon)$ for $\epsilon<a^{U}-a^{L}$.

- $\quad$ Suppose that $\|[0,0]\|=0$. Then $A \in \mathcal{B}^{\diamond}(A ; \epsilon)$, as $A=A \oplus[0,0]$.

Proposition 6. Let $(\mathcal{I},\|\cdot\|)$ be a pseudo-seminormed interval space.

(i) For $A \in \mathcal{I}$ with $\omega_{A}=A \ominus A \in \Omega$, we have $\mathcal{B}(A ; \epsilon) \oplus \omega_{A} \subseteq \mathcal{B}^{\diamond}(A ; \epsilon)$.

(ii) If $\|\cdot\|$ satisfies the null sub-inequality, then $\mathcal{B}^{\diamond}(A ; \epsilon) \subseteq \mathcal{B}(A ; \epsilon)$.

(iii) Suppose that $\|\cdot\|$ satisfies the null sub-inequality. Then, for any $A \in \mathcal{I}$ with $\omega_{A}=A \ominus A \in \Omega$, we have $\mathcal{B}(A ; \epsilon) \oplus \omega_{A} \subseteq \mathcal{B}(A ; \epsilon)$ and $\mathcal{B}^{\diamond}(A ; \epsilon) \oplus \omega_{A} \subseteq \mathcal{B}^{\diamond}(A ; \epsilon)$.

Proof. To prove part (i), for any $B \in \mathcal{B}(A$; $\epsilon)$, i.e., $\|B \ominus A\|<\epsilon$, if we take $C=B \ominus A$, then $\|C\|<\epsilon$ and $A \oplus C=B \oplus \omega_{A}$ by adding $A$ on both sides. This shows the inclusion

$$
\mathcal{B}(A ; \epsilon) \oplus \omega_{A} \subseteq\{A \oplus C:\|C\|<\epsilon\}=\mathcal{B}^{\diamond}(A ; \epsilon) .
$$

To prove part (ii), for $C \in \mathcal{I}$ with $\|C\|<\epsilon$, since $\|\cdot\|$ satisfies the null sub-inequality, it follows that

$$
\|(A \oplus C) \ominus A\|=\|\omega \oplus C\| \leq\|C\|<\epsilon,
$$

which says that $A \oplus \mathcal{C} \in \mathcal{B}(A ; \epsilon)$ and shows the inclusion

$$
\mathcal{B}^{\diamond}(A ; \epsilon)=\{A \oplus C:\|C\|<\epsilon\} \subseteq \mathcal{B}(A ; \epsilon) .
$$

Part (iii) follows from parts (i) and (ii) immediately. This completes the proof.

Proposition 7. Let $(\mathcal{I},\|\cdot\|)$ be a pseudo-seminormed interval space.

(i) If $\|\cdot\|$ satisfies the null superinequality, then $\mathcal{B}(A \oplus \omega ; \epsilon) \subseteq \mathcal{B}(A ; \epsilon)$ for any $\omega \in \Omega$.

(ii) If $\|\cdot\|$ satisfies the null sub-inequality, then we have the following inclusions

$$
\mathcal{B}(A ; \epsilon) \subseteq \mathcal{B}(A \oplus \omega ; \epsilon) \text { and } \mathcal{B}^{\diamond}(A \oplus \omega ; \epsilon) \subseteq \mathcal{B}^{\diamond}(A ; \epsilon)
$$

for any $\omega \in \Omega$.

(iii) If $\|\cdot\|$ satisfies the null equality, then $\mathcal{B}(A \oplus \omega ; \epsilon)=\mathcal{B}(A ; \epsilon)$ for any $\omega \in \Omega$.

Proof. To prove part (i), given any $B \in \mathcal{B}(A \oplus \omega ; \epsilon)$, i.e., $\|(A \oplus \omega) \ominus B\|<\epsilon$, using the null superinequality, we have

$$
\epsilon>\|(A \oplus \omega) \ominus B\|=\|(A \ominus B) \oplus \omega\| \geq\|A \ominus B\|,
$$

which shows $B \in \mathcal{B}(A ; \epsilon)$ and the inclusion $\mathcal{B}(A \oplus \omega ; \epsilon) \subseteq \mathcal{B}(A ; \epsilon)$.

To prove the first inclusion of part (ii), using the null sub-inequality, the inclusion $\mathcal{B}(A ; \epsilon) \subseteq$ $\mathcal{B}(A \oplus \omega ; \epsilon)$ follows from the following expression,

$$
\epsilon>\|A \ominus B\| \geq\|(A \ominus B) \oplus \omega\|=\|(A \oplus \omega) \ominus B\| .
$$

To prove the second inclusion of part (ii), for $B=A \oplus \omega \oplus C \in \mathcal{B}^{\diamond}(A \oplus \omega ; \epsilon)$ with $\|C\|<\epsilon$, let $\bar{C}=\omega \oplus C$. Then, using the null sub-inequality, we have

$$
\|\bar{C}\|=\|\omega \oplus C\| \leq\|C\|<\epsilon,
$$


which says that $B=A \oplus \bar{C} \in \mathcal{B}^{\diamond}(A ; \epsilon)$. Therefore we obtain the inclusion $\mathcal{B}^{\diamond}(A \oplus \omega ; \epsilon) \subseteq \mathcal{B}^{\diamond}(A ; \epsilon)$. Part (iii) follows from parts (i) and (ii) immediately. This completes the proof.

Recall that, in the (conventional) normed space $(X,\|\cdot\|)$, the open ball $\mathcal{B}(x ; \epsilon)$ is given by

$$
\mathcal{B}(x ; \epsilon)=\{y \in X:\|x-y\|<\epsilon\} .
$$

Then, we have the following equality

$$
\mathcal{B}(x ; \epsilon)+\hat{x}=\mathcal{B}(x+\hat{x} ; \epsilon) .
$$

However, in the normed interval space $(\mathcal{I},\|\cdot\|)$, the intuitive observation (3) will not hold true in general. The following proposition presents the exact relationship.

Proposition 8. Let $(\mathcal{I},\|\cdot\|)$ be a pseudo-seminormed interval space.

(i) We have the equality

$$
\mathcal{B}^{\diamond}(A ; \epsilon) \oplus \widehat{A}=\mathcal{B}^{\diamond}(A \oplus \widehat{A} ; \epsilon) .
$$

In particular, for any $\omega \in \Omega$, we also have

$$
\mathcal{B}^{\diamond}(A ; \epsilon) \oplus \omega=\mathcal{B}^{\diamond}(A \oplus \omega ; \epsilon) .
$$

(ii) Suppose that $\|\cdot\|$ satisfies the null sub-inequality. Then, we have the inclusion

$$
\mathcal{B}(A ; \epsilon) \oplus \widehat{A} \subseteq \mathcal{B}(A \oplus \widehat{A} ; \epsilon) .
$$

We further assume that $\|\cdot\|$ satisfies the null equality. Then, for any $\omega \in \Omega$, we also have the inclusions

$$
\mathcal{B}(A ; \epsilon) \oplus \omega \subseteq \mathcal{B}(A ; \epsilon) \text { and } \mathcal{B}(\omega ; \epsilon) \oplus \widehat{A} \subseteq \mathcal{B}(\widehat{A} ; \epsilon) .
$$

(iii) Suppose that $\|\cdot\|$ satisfies the null sub-inequality. For any $A \in \mathcal{I}$ with $\omega_{A}=A \ominus A$, we have the inclusion

$$
\mathcal{B}(A ; \epsilon) \oplus \omega_{A} \subseteq A \oplus \mathcal{B}\left(\omega_{A} ; \epsilon\right) .
$$

(iv) For any $\widehat{A} \in \mathcal{I}$ with $\omega_{\widehat{A}}=\widehat{A} \ominus \widehat{A}$, we have the inclusion

$$
\mathcal{B}(A \oplus \widehat{A} ; \epsilon) \oplus \omega_{\widehat{A}} \subseteq \mathcal{B}(A ; \epsilon) \oplus \widehat{A} .
$$

Proof. Part (i) follows from the following equality

$$
(A \oplus C) \oplus \widehat{A}=(A \oplus \widehat{A}) \oplus C \text { for }\|C\|<\epsilon .
$$

To prove part (ii), for $B \in \mathcal{B}(A ; \epsilon) \oplus \widehat{A}$, we have $B=\widehat{B} \oplus \widehat{A}$ with $\|A \ominus \widehat{B}\|<\epsilon$. Then, by (1) and the null sub-inequality, we can obtain

$$
\|(A \oplus \widehat{A}) \ominus B\|=\|(A \oplus \widehat{A}) \ominus(\widehat{B} \oplus \widehat{A})\|=\|(A \ominus \widehat{B}) \oplus(\widehat{A} \ominus \widehat{A})\| \leq\|A \ominus \widehat{B}\|<\epsilon,
$$

which says that $B \in \mathcal{B}(A \oplus \widehat{A} ; \epsilon)$. Therefore, we obtain the inclusion $\mathcal{B}(A ; \epsilon) \oplus \widehat{A} \subseteq \mathcal{B}(A \oplus \widehat{A} ; \epsilon)$. Now, we take $\widehat{A}=\omega$. By part (iii) of Proposition 7, we have

$$
\mathcal{B}(A ; \epsilon) \oplus \omega \subseteq \mathcal{B}(A \oplus \omega ; \epsilon)=\mathcal{B}(A ; \epsilon) .
$$


Similarly, if we take $A=\omega$, then we have

$$
\mathcal{B}(\omega ; \epsilon) \oplus \widehat{A} \subseteq \mathcal{B}(\omega \oplus \widehat{A} ; \epsilon)=\mathcal{B}(\widehat{A} ; \epsilon) .
$$

To prove part (iii), for $\widehat{A} \in \mathcal{B}(A ; \epsilon)$, we have $\widehat{A} \oplus \omega_{A}=A \oplus(\widehat{A} \ominus A)$. The null sub-inequality gives

$$
\left\|\omega_{A} \ominus(\widehat{A} \ominus A)\right\| \leq\|\widehat{A} \ominus A\|<\epsilon,
$$

which says that $\widehat{A} \ominus A \in \mathcal{B}(\omega ; \epsilon)$, i.e.,

$$
\widehat{A} \oplus \omega_{A}=A \oplus(\widehat{A} \ominus A) \in A \oplus \mathcal{B}\left(\omega_{A} ; \epsilon\right) .
$$

To prove part (iv), for $B \in \mathcal{B}(A \oplus \widehat{A} ; \epsilon)$, we have $\|B \ominus(A \oplus \widehat{A})\|<\epsilon$. By Equation (1), we also have

$$
\epsilon>\|B \ominus(A \oplus \widehat{A})\|=\|(B \ominus \widehat{A}) \ominus A\| .
$$

This shows that $B \ominus \widehat{A} \in \mathcal{B}(A ; \epsilon)$. Let $\omega_{\widehat{A}}=\widehat{A} \ominus \widehat{A} \in \Omega$. Since $B \oplus \omega_{\widehat{A}}=(B \ominus \widehat{A}) \oplus \widehat{A}$, it says that $B \oplus \omega_{\widehat{A}} \in \mathcal{B}(A ; \epsilon) \oplus \widehat{A}$. In other words, we have the inclusion

$$
\mathcal{B}(A \oplus \widehat{A} ; \epsilon) \oplus \omega_{\widehat{A}} \subseteq \mathcal{B}(A ; \epsilon) \oplus \widehat{A} .
$$

This completes the proof.

Proposition 9. Let $(\mathcal{I},\|\cdot\|)$ be a pseudo-seminormed interval space.

(i) The following statements hold true.

- Suppose that $\|\cdot\|$ satisfies the null superinequality. For any $\omega \in \Omega$, if $A \oplus \omega \in \mathcal{B}\left(A_{0} ; \epsilon\right)$, then $A \in \mathcal{B}\left(A_{0} ; \epsilon\right)$.

- Suppose that $\|\cdot\|$ satisfies the null sub-inequality. For any $\omega \in \Omega$, if $A \in \mathcal{B}\left(A_{0} ; \epsilon\right)$, then $A \oplus \omega \in \mathcal{B}\left(A_{0} ; \epsilon\right)$, and if $A \in \mathcal{B}^{\diamond}\left(A_{0} ; \epsilon\right)$, then $A \oplus \omega \in \mathcal{B}^{\diamond}\left(A_{0} ; \epsilon\right)$.

- Suppose that $\|\cdot\|$ satisfies the null equality. Then, for any $\omega \in \Omega, A \oplus \omega \in \mathcal{B}\left(A_{0} ; \epsilon\right)$ if and only if $A \in \mathcal{B}\left(A_{0} ; \epsilon\right)$.

(ii) We have the inclusions

$$
\mathcal{B}(A ; \epsilon) \subseteq \mathcal{B}(A ; \epsilon) \oplus \Omega \text { and } \mathcal{B}^{\diamond}(A ; \epsilon) \subseteq \mathcal{B}^{\diamond}(A ; \epsilon) \oplus \Omega
$$

If we further assume that $\|\cdot\|$ satisfies the null sub-inequality, then

$$
\mathcal{B}(A ; \epsilon) \oplus \Omega=\mathcal{B}(A ; \epsilon) \text { and } \mathcal{B}^{\diamond}(A ; \epsilon) \oplus \Omega=\mathcal{B}^{\diamond}(A ; \epsilon) \text {. }
$$

(iii) Suppose that $\|\cdot\|$ satisfies the null condition. Given a fixed $\omega \in \Omega$, we have $\Omega \oplus \omega \subseteq \mathcal{B}^{\diamond}(\omega ; \epsilon)$ and $\Omega \subseteq \mathcal{B}(\omega ; \epsilon)$.

(iv) Given any fixed $\omega \in \Omega$ and $\alpha \neq 0$, we have the following results.

- Suppose that $\|\cdot\|$ satisfies the null equality. Then $\alpha \mathcal{B}(\omega ; \epsilon) \subseteq \mathcal{B}(\omega ;|\alpha| \epsilon)$.

- Suppose that $\|\cdot\|$ satisfies the null superinequality, and that $\alpha<1$. Then $\mathcal{B}(\omega ;|\alpha| \epsilon) \subseteq \alpha \mathcal{B}(\omega ; \epsilon)$.

(v) Given any fixed $\omega \in \Omega$ and $\alpha \neq 0$, we have

$$
\alpha \mathcal{B}^{\diamond}(\omega ; \epsilon) \subseteq \mathcal{B}^{\diamond}(\alpha \omega ;|\alpha| \epsilon) \text { and } \mathcal{B}^{\diamond}(\alpha \omega ;|\alpha| \epsilon) \subseteq \alpha \mathcal{B}^{\diamond}(\omega ; \epsilon) \text {. }
$$


Proof. The first case of part (i) follows from the following expression,

$$
\left\|A \ominus A_{0}\right\| \leq\left\|(A \oplus \omega) \ominus A_{0}\right\|<\epsilon .
$$

The second case of part (i) regarding the open ball $\mathcal{B}\left(A_{0} ; \epsilon\right)$ follows from the following expression,

$$
\left\|(A \oplus \omega) \ominus A_{0}\right\| \leq\left\|A \ominus A_{0}\right\|<\epsilon .
$$

For the open ball $\mathcal{B}^{\diamond}\left(A_{0} ; \epsilon\right)$, if $A \in \mathcal{B}^{\diamond}\left(A_{0} ; \epsilon\right)$, then $A=A_{0} \oplus C$ with $\|C\|<\epsilon$. Given an $\omega \in \Omega$, let $\bar{C}=C \oplus \omega$. Therefore, we have $A \oplus \omega=A_{0} \oplus \bar{C}$, where

$$
\|\bar{C}\|=\|C \oplus \omega\| \leq\|C\|<\epsilon,
$$

which says that $A \oplus \omega \in \mathcal{B}^{\diamond}\left(A_{0} ; \epsilon\right)$. The third case of part (i) follows from the previous two cases.

To prove part (ii), as $[0,0] \in \Omega$ and $B=B \oplus[0,0]$, we have the inclusions $\mathcal{B}(A ; \epsilon) \subseteq \mathcal{B}(A ; \epsilon) \oplus \Omega$ and $\mathcal{B}^{\diamond}(A ; \epsilon) \subseteq \mathcal{B}^{\diamond}(A ; \epsilon) \oplus \Omega$. On the other hand, for $A \in \mathcal{B}\left(A_{0} ; \epsilon\right)$ and $\omega \in \Omega$, from (4), we see that $A \oplus \omega \in \mathcal{B}\left(A_{0} ; \epsilon\right)$, which shows the inclusion $\mathcal{B}\left(A_{0} ; \epsilon\right) \oplus \Omega \subseteq \mathcal{B}\left(A_{0} ; \epsilon\right)$. Also, for $B=A \oplus C \in$ $\mathcal{B}^{\diamond}(A ; \epsilon)$ with $\|C\|<\epsilon$, let $\bar{C}=\omega \oplus C$. By Equation (5), we have $B \oplus \omega=A \oplus \bar{C} \in \mathcal{B}^{\diamond}(A ; \epsilon)$, which shows the inclusion $\mathcal{B}^{\diamond}(A ; \epsilon) \oplus \Omega \subseteq \mathcal{B}^{\diamond}(A ; \epsilon)$. This proves part (ii).

To prove part (iii), for any $\omega^{\prime} \in \Omega$, we have $\left\|\omega^{\prime}\right\|=0$, which says that $\omega \oplus \omega^{\prime} \in \mathcal{B}^{\diamond}(\omega ; \epsilon)$. Therefore, we obtain the inclusion $\Omega \oplus \omega \subseteq \mathcal{B}^{\diamond}(\omega ; \epsilon)$. On the other hand, we also have

$$
\left\|\omega^{\prime} \ominus \omega\right\|=\left\|\omega^{\prime} \oplus(-\omega)\right\| \leq\left\|\omega^{\prime}\right\|+\|-\omega\|=\left\|\omega^{\prime}\right\|+\|\omega\|=0,
$$

which shows that $\omega^{\prime} \in \mathcal{B}(\omega ; \epsilon)$, i.e., $\Omega \subseteq \mathcal{B}(\omega ; \epsilon)$.

To prove the first case of part (iv), for $A \in \mathcal{B}(\omega ; \epsilon)$, as $\alpha \omega \in \Omega$, we have

$$
\|\omega \ominus \alpha A\|=\|(\omega \oplus \alpha \omega) \ominus \alpha A\|=\|\alpha \omega \ominus \alpha A\|=\|\alpha(A \ominus \omega)\|=|\alpha|\|A \ominus \omega\|<|\alpha| \epsilon,
$$

i.e., $\alpha A \in \mathcal{B}(\omega ;|\alpha| \epsilon)$. This shows the inclusion $\alpha \mathcal{B}(\omega ; \epsilon) \subseteq \mathcal{B}(\omega ;|\alpha| \epsilon)$. To prove the second case of part (v), for $A \in \mathcal{B}(\omega ;|\alpha| \epsilon)$, we have $\|\omega \ominus A\|<|\alpha| \epsilon$, i.e.,

$$
\|(\omega / \alpha) \ominus(A / \alpha)=\|(\omega \ominus A) / \alpha\|=1 /|\alpha| \cdot\| \omega \ominus A \|<\epsilon .
$$

As $\alpha<1$, from part (vi) of Proposition 3, we have $\omega / \alpha=\omega \oplus \widehat{\omega}$ for some $\widehat{\omega} \in \Omega$. Therefore, we obtain

$$
\|\omega \ominus(A / \alpha)\| \leq\|(\omega \oplus \widehat{\omega}) \ominus(A / \alpha)\|=\|(\omega / \alpha) \ominus(A / \alpha)\|<\epsilon,
$$

which shows that $A / \alpha \in \mathcal{B}(\omega ; \epsilon)$. Therefore we obtain $A \in \alpha \mathcal{B}(\omega ; \epsilon)$, which shows the inclusion $\mathcal{B}(\omega ;|\alpha| \epsilon) \subseteq \alpha \mathcal{B}(\omega ; \epsilon)$.

To prove the first inclusion of part (v), for $A \in \mathcal{B}(\omega ; \epsilon)$, we have $A=\omega \oplus C$ with $\|C\|<\epsilon$. It follows that $\alpha A=\alpha \omega \oplus \alpha C$. Let $\bar{C}=\alpha C$. Then $\|\bar{C}\|<|\alpha| \epsilon$, which shows the inclusion $\alpha \mathcal{B}^{\diamond}(\omega ; \epsilon) \subseteq$ $\mathcal{B}^{\diamond}(\alpha \omega ;|\alpha| \epsilon)$. To prove the second inclusion of part (v), for $A \in \mathcal{B}^{\diamond}(\alpha \omega ;|\alpha| \epsilon)$, we have $A=\alpha \omega \oplus C$ with $\|C\|<|\alpha| \epsilon$. Let $\widehat{C}=C / \alpha$. Then

$$
A=\alpha \omega \oplus C=\alpha \omega \oplus \alpha(C / \alpha)=\alpha \omega \oplus \alpha \widehat{C}=\alpha(\omega \oplus \widehat{C}) \text { with }\|\widehat{C}\|<\epsilon,
$$

which says that $A \in \alpha \mathcal{B}^{\diamond}(\omega ; \epsilon)$. This completes the proof.

\section{Open Sets}

Let $(\mathcal{I},\|\cdot\|)$ be a pseudo-seminormed interval space. We are going to consider the open subsets of $\mathcal{I}$. 
Definition 4. Let $(\mathcal{I},\|\cdot\|)$ be a pseudo-seminormed interval space, and let $\mathcal{A}$ be a nonempty subset of $\mathcal{I}$.

- A point, $A_{0} \in \mathcal{A}$, is said to be a interior point of $\mathcal{A}$ if and only if there exists $\epsilon>0$, such that $\mathcal{B}\left(A_{0} ; \epsilon\right) \subseteq \mathcal{A}$. The collection of all interior points of $\mathcal{A}$ is called the interior of $\mathcal{A}$ and is denoted by $\operatorname{int}(\mathcal{A})$.

- A point, $A_{0} \in \mathcal{A}$, is said to be a type-I-interior point of $\mathcal{A}$ if and only if there exists $\epsilon>0$, such that $\mathcal{B}\left(A_{0} ; \epsilon\right) \oplus \Omega \subseteq \mathcal{A}$. The collection of all type-I-interior points of $\mathcal{A}$ is called the type-I-interior of $\mathcal{A}$ and is denoted by int $t^{(I)}(\mathcal{A})$.

- A point, $A_{0} \in \mathcal{A}$, is said to be a type-II-interior point of $\mathcal{A}$ if and only if there exists $\epsilon>0$, such that $\mathcal{B}\left(A_{0} ; \epsilon\right) \subseteq \mathcal{A} \oplus \Omega$. The collection of all type-II-interior points of $\mathcal{A}$ is called the type-II-interior of $\mathcal{A}$ and is denoted by int ${ }^{(I I)}(\mathcal{A})$.

- A point, $A_{0} \in \mathcal{A}$, is said to be a type-III-interior point of $\mathcal{A}$ if and only if there exists $\epsilon>0$, such that $\mathcal{B}\left(A_{0} ; \epsilon\right) \oplus \Omega \subseteq \mathcal{A} \oplus \Omega$. The collection of all type-III-interior points of $\mathcal{A}$ is called the type-III-interior of $\mathcal{A}$ and is denoted by int ${ }^{(I I)}(\mathcal{A})$.

The different types of $\diamond$-interior points based on the open ball $\mathcal{B}^{\diamond}\left(A_{0} ; \epsilon\right)$ can be similarly defined. For example, int ${ }^{(\text {oIII) }}(\mathcal{A})$ denotes the $\diamond$-type-III-interior of $\mathcal{A}$.

Remark 3. Recall that we cannot have the property $A \in \mathcal{B}(A ; \epsilon)$ in general by Remark 2, unless $\|\cdot\|$ satisfies the null condition. Given any $A \in \mathcal{I}$ with $\|A \ominus A\| \neq 0$, it follows that $A \notin \mathcal{B}\left(A ; \epsilon^{*}\right)$ for $\epsilon^{*}<\|A \ominus A\|$. Now, given $\epsilon<\epsilon^{*}$, it is clear that $\mathcal{B}(A ; \epsilon) \subseteq \mathcal{B}\left(A ; \epsilon^{*}\right)$. Let use take $\mathcal{A}=\mathcal{B}\left(A ; \epsilon^{*}\right)$. It means that the open ball $\mathcal{B}(A ; \epsilon)$ is contained in $\mathcal{A}$ even though the center $A$ is not in $\mathcal{A}$.

Remark 4. From Remark 3 , it is possible that there exists an open ball such that $\mathcal{B}(A ; \epsilon)$ is contained in $\mathcal{A}$, even though the center $A$ is not in $\mathcal{A}$. In this situation, we will not say that $A$ is an interior point, since $A$ is not in $\mathcal{A}$. Also, the sets $\mathcal{B}(A ; \epsilon) \oplus \Omega$ and $\mathcal{B}^{\diamond}(A ; \epsilon) \oplus \Omega$ will not necessarily contain the center $A$. In other words, it is possible that there exists an open ball such that $\mathcal{B}(A ; \epsilon) \oplus \Omega$ is contained in $\mathcal{A}$, even though the center $A$ is not in $\mathcal{A}$. In this situation, we will not say that $A$ is an type-I-interior point, as $A$ is not in $\mathcal{A}$. However, we have the following observations.

- Suppose that $\|\cdot\|$ satisfies the null condition. Then $A \in \mathcal{B}(A ; \epsilon)$. Since $A=A \oplus \theta$, we also have $A \in \mathcal{B}(A ; \epsilon) \oplus \Omega$.

- Suppose that $\|[0,0]\|=0$. The second observation of Remark 2 says that $A \in \mathcal{B}^{\diamond}(A ; \epsilon)$. As $A=A \oplus \theta$, it follows that $A \in \mathcal{B}^{\diamond}(A ; \epsilon) \oplus \Omega$.

Inspired by Remark 4, we can define the different concepts of pseudo-interior point.

Definition 5. Let $(\mathcal{I},\|\cdot\|)$ be a pseudo-seminormed interval space, and let $\mathcal{A}$ be a nonempty subset of $\mathcal{I}$.

- A point $A_{0} \in \mathcal{I}$ is said to be a pseudo-interior point of $\mathcal{A}$ if and only if there exists $\epsilon>0$ such that $\mathcal{B}\left(A_{0} ; \epsilon\right) \subseteq \mathcal{A}$. The collection of all pseudo-interior points of $\mathcal{A}$ is called the pseudo-interior of $\mathcal{A}$ and is denoted by $\operatorname{pint}(\mathcal{A})$.

- A point $A_{0} \in \mathcal{I}$ is said to be a type-I-pseudo-interior point of $\mathcal{A}$ if and only if there exists $\epsilon>0$ such that $\mathcal{B}\left(A_{0} ; \epsilon\right) \oplus \Omega \subseteq \mathcal{A}$. The collection of all type-I-pseudo-interior points of $\mathcal{A}$ is called the type-I-pseudo-interior of $\mathcal{A}$ and is denoted by $\operatorname{pint}^{(I)}(\mathcal{A})$.

- A point $A_{0} \in \mathcal{I}$ is said to be a type-II-pseudo-interior point of $\mathcal{A}$ if and only if there exists $\epsilon>0$ such that $\mathcal{B}\left(A_{0} ; \epsilon\right) \subseteq \mathcal{A} \oplus \Omega$. The collection of all type-II-pseudo-interior points of $\mathcal{A}$ is called the type-II-pseudo-interior of $\mathcal{A}$ and is denoted by pint ${ }^{(I I)}(\mathcal{A})$.

- A point $A_{0} \in \mathcal{I}$ is said to be a type-III-pseudo-interior point of $\mathcal{A}$ if and only if there exists $\epsilon>0$ such that $\mathcal{B}\left(A_{0} ; \epsilon\right) \oplus \Omega \subseteq \mathcal{A} \oplus \Omega$. The collection of all type-III-pseudo-interior points of $\mathcal{A}$ is called the of $\mathcal{A}$ and is denoted by $\operatorname{pint}^{(I I I)}(\mathcal{A})$.

The different types of $\diamond$-pseudo-interior point based on the open ball $\mathcal{B}^{\diamond}\left(A_{0} ; \epsilon\right)$ can be similarly defined. 
Remark 5. Note that the difference between Definitions 4 and 5 is that we consider $A_{0} \in \mathcal{A}$ in Definition 4 and $A_{0} \in \mathcal{I}$ in Definition 5. From Remark 3, if $\epsilon^{*}<\|A \ominus A\|$, then $A$ is a pseudo-interior point of $\mathcal{B}\left(A ; \epsilon^{*}\right)$. We also have the following observations.

- It is clear that $\operatorname{int}(\mathcal{A}) \subseteq \operatorname{pint}(\mathcal{A}), \operatorname{int}^{(I)}(\mathcal{A}) \subseteq \operatorname{pint}^{(I)}(\mathcal{A}), \operatorname{int}^{(I I)}(\mathcal{A}) \subseteq \operatorname{pint}^{(I I)}(\mathcal{A})$ and int $t^{(I I)}(\mathcal{A}) \subseteq$ $\operatorname{pint}^{(I I I)}(\mathcal{A})$. The same inclusions can also apply to the different types of $\diamond$-interior and $\diamond$-pseudo-interior.

- It is clear that $\operatorname{int}(\mathcal{A}) \subseteq \mathcal{A}$, $\operatorname{int}^{(I)}(\mathcal{A}) \subseteq \mathcal{A}$, int $t^{(I I)}(\mathcal{A}) \subseteq \mathcal{A}$ and int ${ }^{(I I I)}(\mathcal{A}) \subseteq \mathcal{A}$. However, those kinds of inclusions cannot hold true for the pseudo-interior.

- From Remark 2, we have the following observations.

- Suppose that $\|\cdot\|$ satisfies the null condition. Then the concepts of interior point and the concepts of pseudo-interior point are equivalent, since $A_{0}$ is in the open ball $\mathcal{B}\left(A_{0} ; \epsilon\right)$.

- Suppose that $\|\theta\|=0$. Then the concepts of $\diamond$-type of interior point and the concepts of $\diamond$-type of pseudo-interior point are equivalent, since $A_{0}$ is in the open ball $\mathcal{B}^{\diamond}\left(A_{0} ; \epsilon\right)$.

Example 4. The non-negative real-valued function $\|\cdot\|$ on $\mathcal{I}$ is defined by

$$
\left\|\left[a^{L}, a^{U}\right]\right\|=a^{U}-a^{L} .
$$

Then, $(\mathcal{I},\|\cdot\|)$ is a seminormed interval, space such that the null condition is not satisfied. The open balls $\mathcal{B}\left(\left[a_{0}^{L}, a_{0}^{U}\right] ; \epsilon\right)$ and $\mathcal{B}^{\diamond}\left(\left[a_{0}^{L}, a_{0}^{U}\right] ; \epsilon\right)$ with radius $\epsilon$ are given by

$$
\begin{aligned}
\mathcal{B}\left(\left[a_{0}^{L}, a_{0}^{U}\right] ; \epsilon\right) & =\left\{\left[a^{L}, a^{U}\right] \in \mathcal{I}:\left\|\left[a_{0}^{L}, a_{0}^{U}\right] \ominus\left[a^{L}, a^{U}\right]\right\|<\epsilon\right\} \\
& =\left\{\left[a^{L}, a^{U}\right] \in \mathcal{I}: a_{0}^{U}-a_{0}^{L}+a^{U}-a^{L}<\epsilon\right\}
\end{aligned}
$$

and

$$
\begin{aligned}
\mathcal{B}^{\diamond}\left(\left[a_{0}^{L}, a_{0}^{U}\right] ; \epsilon\right) & =\left\{\left[a_{0}^{L}, a_{0}^{U}\right] \oplus\left[a^{L}, a^{U}\right] \in \mathcal{I}:\left\|\left[a^{L}, a^{U}\right]\right\|<\epsilon\right\} \\
& =\left\{\left[a_{0}^{L}+a^{L}, a_{0}^{U}+a^{U}\right] \in \mathcal{I}: a^{U}-a^{L}<\epsilon\right\} .
\end{aligned}
$$

- $\quad$ Suppose that $\epsilon \leq 2\left(a_{0}^{U}-a_{0}^{L}\right)$. As

$$
\left[a_{0}^{L}, a_{0}^{U}\right] \ominus\left[a_{0}^{L}, a_{0}^{U}\right]=2\left(a_{0}^{U}-a_{0}^{L}\right) \geq \epsilon,
$$

it says that $\left[a_{0}^{L}, a_{0}^{U}\right] \notin \mathcal{B}\left(\left[a_{0}^{L}, a_{0}^{U}\right] ; \epsilon\right)$.

- Given any $\epsilon>0$, we see that $\left[a_{0}^{L}, a_{0}^{U}\right] \in \mathcal{B}^{\diamond}\left(\left[a_{0}^{L}, a_{0}^{U}\right] ; \epsilon\right)$, as $\left[a^{L}, a^{U}\right]=[0,0] \in \mathcal{I}$.

We consider the following set

$$
A=\left\{\left[a^{L}, a^{U}\right] \in \mathcal{I}: a^{U}-a^{L} \leq 5\right\}
$$

For $\left[a_{0}^{L}, a_{0}^{U}\right]=[0,6]$, we see that $\left[a_{0}^{L}, a_{0}^{U}\right] \notin \mathcal{A}$. For the open ball $\mathcal{B}\left(\left[a_{0}^{L}, a_{0}^{U}\right] ; \epsilon\right)$ with radius $\epsilon=8$, we see that

$$
\begin{aligned}
\mathcal{B}\left(\left[a_{0}^{L}, a_{0}^{U}\right] ; \epsilon\right) & =\mathcal{B}([0,6] ; 8)=\left\{\left[a^{L}, a^{U}\right] \in \mathcal{I}: 6+a^{U}-a^{L}<8\right\} \\
& =\left\{\left[a^{L}, a^{U}\right] \in \mathcal{I}: a^{U}-a^{L}<2\right\} \subset A
\end{aligned}
$$

which says that $\left[a_{0}^{L}, a_{0}^{U}\right]=[0,6]$ is a pseudo-interior point of $\mathcal{A}$. 
Remark 6. From part (ii) of Proposition 9, if $\|\cdot\|$ satisfies the null sub-inequality, then the concepts of interior point and type-I-interior point are equivalent and the concepts of type-II-interior point and type-III-interior point are equivalent. The same situation also applies to the cases of pseudo-interior points.

Remark 7. Suppose that $\|\cdot\|$ satisfies the null sub-inequality. From part (ii) of Proposition 6, we see that if $A_{0}$ is an interior (resp. type-I-interior, type-II-interior, type-III-interior) point then it is also an $\diamond$-interior (resp.

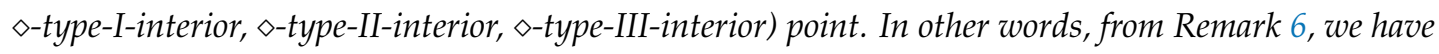

$$
\operatorname{int}(\mathcal{A})=\operatorname{int} t^{(I)}(\mathcal{A}) \subseteq \operatorname{int} t^{(\diamond I)}(\mathcal{A})=\operatorname{int} t^{\diamond}(\mathcal{A})
$$

and

$$
\operatorname{int} t^{(I I)}(\mathcal{A})=\operatorname{int} t^{(I I I)}(\mathcal{A}) \subseteq \operatorname{int} t^{(\diamond I I I)}(\mathcal{A})=\operatorname{int} t^{(\diamond I I)}(\mathcal{A}) .
$$

Regarding the different concepts of pseudo-interior point, we also have

$$
\operatorname{pint}(\mathcal{A})=\operatorname{pint}^{(I)}(\mathcal{A}) \subseteq \operatorname{pint}^{(\odot l)}(\mathcal{A})=\operatorname{pint} t^{\diamond}(\mathcal{A})
$$

and

$$
\operatorname{pint}^{(I I)}(\mathcal{A})=\operatorname{pint}^{(I I I)}(\mathcal{A}) \subseteq \operatorname{pint}^{(\diamond I I I)}(\mathcal{A})=\operatorname{pint}^{(\diamond I I)}(\mathcal{A})
$$

Remark 8. Let $(\mathcal{I},\|\cdot\|)$ be a pseudo-seminormed interval space.

- Suppose that the center $A_{0}$ is in the open ball $\mathcal{B}\left(A_{0} ; \epsilon\right)$. Then, the concepts of interior point and pseudo-interior point are equivalent. It follows that $\operatorname{pint}(\mathcal{A})=\operatorname{int}(\mathcal{A}) \subseteq \mathcal{A}$. Similarly, if the center $A_{0}$ is in the open ball $\mathcal{B}^{\diamond}\left(A_{0} ; \epsilon\right)$, then $\operatorname{pint}^{\diamond}(\mathcal{A})=\operatorname{int}^{\diamond}(\mathcal{A}) \subseteq \mathcal{A}$.

- $\quad$ From part (ii) of Proposition 9, we have $\mathcal{B}(A ; \epsilon) \subseteq \mathcal{B}(A ; \epsilon) \oplus \Omega$ and $\mathcal{B}^{\diamond}(A ; \epsilon) \subseteq \mathcal{B}^{\diamond}(A ; \epsilon) \oplus \Omega$. Suppose that the center $A_{0}$ is in the open ball $\mathcal{B}\left(A_{0} ; \epsilon\right)$. Let $A_{0}$ be an type-I-pseudo-interior point of $\mathcal{A}$. As

$$
A_{0} \in \mathcal{B}\left(A_{0} ; \epsilon\right) \subseteq \mathcal{B}\left(A_{0} ; \epsilon\right) \oplus \Omega \subseteq \mathcal{A}
$$

using Remark 5, we obtain

$$
\operatorname{pint}^{(I)}(\mathcal{A}) \subseteq \operatorname{int}(\mathcal{A}) \subseteq \mathcal{A} \text { and } \operatorname{pint}^{(I)}(\mathcal{A}) \subseteq \operatorname{int}^{(I)}(\mathcal{A}) \subseteq \operatorname{pint}^{(I)}(\mathcal{A})
$$

which also implies pint ${ }^{(I)}(\mathcal{A})=\operatorname{int}^{(I)}(\mathcal{A})$. Similarly, if the center $A_{0}$ is in the open ball $\mathcal{B}^{\diamond}\left(A_{0} ; \epsilon\right)$, then $\operatorname{pint}^{(\diamond I)}(\mathcal{A})=\operatorname{int}^{(\diamond I)}(\mathcal{A})$.

- $\quad$ Suppose that $\mathcal{A} \oplus \Omega \subseteq \mathcal{A}$. We have the following observations. Assume that the center $A_{0}$ is in the open ball $\mathcal{B}\left(A_{0} ; \epsilon\right)$. Let $A_{0}$ be an type-II-pseudo-interior point of $\mathcal{A}$. As

$$
A_{0} \in \mathcal{B}\left(A_{0} ; \epsilon\right) \subseteq \mathcal{A} \oplus \Omega \subseteq \mathcal{A}
$$

we obtain

$$
\operatorname{pint}^{(I I)}(\mathcal{A}) \subseteq \operatorname{int}(\mathcal{A}) \subseteq \mathcal{A} \text { and } \operatorname{pint}(I I)(\mathcal{A}) \subseteq \operatorname{int}(I I)(\mathcal{A}) \subseteq \operatorname{pint}(I I)(\mathcal{A}),
$$

which also implies pint ${ }^{(I I)}(\mathcal{A})=\operatorname{int}^{(I I)}(\mathcal{A})$. Similarly, if the center $A_{0}$ is in the open ball $\mathcal{B}^{\diamond}\left(A_{0} ; \epsilon\right)$, then $\operatorname{pint}^{(\diamond I)}(\mathcal{A})=\operatorname{int}^{(\diamond I I)}(\mathcal{A})$.

- Suppose that $\mathcal{A} \oplus \Omega \subseteq \mathcal{A}$, we have the following observations. From part (ii) of Proposition 9, we have $\mathcal{B}(A ; \epsilon) \subseteq \mathcal{B}(A ; \epsilon) \oplus \Omega$ and $\mathcal{B}^{\diamond}(A ; \epsilon) \subseteq \mathcal{B}^{\diamond}(A ; \epsilon) \oplus \Omega$. Assume that the center $A_{0}$ is in the open ball $\mathcal{B}\left(A_{0} ; \epsilon\right)$. Let $A_{0}$ be an type-III-pseudo-interior point of $\mathcal{A}$. As

$$
A_{0} \in \mathcal{B}\left(A_{0} ; \epsilon\right) \subseteq \mathcal{B}\left(A_{0} ; \epsilon\right) \oplus \Omega \subseteq \mathcal{A} \oplus \Omega \subseteq \mathcal{A},
$$

we obtain

$$
\operatorname{pint}^{(I I I)}(\mathcal{A}) \subseteq \operatorname{int}(\mathcal{A}) \subseteq \mathcal{A} \text { and } \operatorname{pint} t^{(I I I)}(\mathcal{A}) \subseteq \operatorname{int} t^{(I I I)}(\mathcal{A}) \subseteq \operatorname{pint}{ }^{(I I I)}(\mathcal{A})
$$


which also implies pint $t^{(I I)}(\mathcal{A})=\operatorname{int}^{(I I I)}(\mathcal{A})$. Similarly, if the center $A_{0}$ is in the open ball $\mathcal{B}^{\diamond}\left(A_{0} ; \epsilon\right)$, then $\operatorname{pint}^{(\text {oIII) }}(\mathcal{A})=\operatorname{int}^{(\text {oIII) }}(\mathcal{A})$.

Definition 6. Let $(\mathcal{I},\|\cdot\|)$ be a pseudo-seminormed interval space, and let $\mathcal{A}$ be a nonempty subset of $\mathcal{I}$. The set $\mathcal{A}$ is said to be open if and only if $\mathcal{A}=\operatorname{int}(\mathcal{A})$. The set $\mathcal{A}$ is said to be type-I-open if and only if $\mathcal{A}=\operatorname{int} t^{(I)}(\mathcal{A})$. The set $\mathcal{A}$ is said to be type-II-open if and only if $\mathcal{A}=\operatorname{int}^{(I I)}(\mathcal{A})$. The set $\mathcal{A}$ is said to be type-III-open if and only if $\mathcal{A}=$ int ${ }^{(I I)}(\mathcal{A})$. We can similarly define the $\diamond$-open set based on the $\diamond$-interior. Also, the pseudo-openness can be similarly defined.

We adopt the convention $\varnothing \oplus \Omega=\varnothing$.

Remark 9. Let $(\mathcal{I},\|\cdot\|)$ be a pseudo-seminormed interval space, and let $\mathcal{A}$ be a nonempty subset of $\mathcal{I}$. We consider the extreme cases of the empty set $\varnothing$ and whole set $\mathcal{I}$.

- As the empty set $\varnothing$ contains no elements, $\varnothing$ is open and pseudo-open (we can regard the empty set as an open ball). It is clear that $\mathcal{I}$ is also open and pseudo-open, since $A \in \mathcal{B} \subseteq X$ for any open ball $\mathcal{B}$, i.e., $\mathcal{I} \subseteq \operatorname{int}(\mathcal{I})$ and $\mathcal{I} \subseteq \operatorname{pint}(\mathcal{I})$.

- As $\varnothing \oplus \Omega=\varnothing \subseteq \varnothing$, the empty set $\varnothing$ is type-I-open and type-I-pseudo-open. It is clear that $\mathcal{I}$ is also type-I-open and type-I-pseudo-open, since $A \in \mathcal{B} \oplus \Omega \subseteq X$ for any open ball $\mathcal{B}$, i.e., $\mathcal{I} \subseteq$ int $^{(I)}(\mathcal{I})$ and $\mathcal{I} \subseteq \operatorname{pint}^{(I)}(\mathcal{I})$.

- As $\varnothing \subseteq \varnothing=\Omega \oplus \varnothing, \varnothing$ is type-II-open and type-II-pseudo-open. We also see that $\mathcal{I}$ is an type-II-open and type-II-pseudo-open set, since, for any $A \in \mathcal{I}$ and any open ball $\mathcal{B}$, we have $A \in \mathcal{B} \subseteq \mathcal{I} \subseteq \mathcal{I} \oplus \Omega$ by part (i) of Proposition 3, i.e., $\mathcal{I} \subseteq$ int $^{(I I)}(\mathcal{I})$ and $\mathcal{I} \subseteq \operatorname{pint}^{\text {(II) }}(\mathcal{I})$.

- As $\varnothing \oplus \Omega \subseteq \Omega \oplus \varnothing, \varnothing$ is type-III-open and type-III-pseudo-open. Now, for any $A \in \mathcal{I}$ and any open ball $\mathcal{B}$, we have $A \in \mathcal{B} \subseteq X$, which says that $\mathcal{B} \oplus \Omega \subseteq X \oplus \Omega$, i.e., $\mathcal{I} \subseteq$ int $t^{(I I I)}(\mathcal{I})$ and $\mathcal{I} \subseteq$ pint ${ }^{(I I I)}(\mathcal{I})$. This shows that $\mathcal{I}$ is type-III-open and type-III-pseudo-open.

We have the above similar results for the types of $\diamond$-open sets and $\diamond$-pseudo-open sets.

Proposition 10. Let $(\mathcal{I},\|\cdot\|)$ be a pseudo-seminormed interval space, and let $\mathcal{A}$ be a nonempty subset of $\mathcal{I}$.

- If $\mathcal{A}$ is pseudo-open, i.e., $\mathcal{A}=\operatorname{pint}(\mathcal{A})$, then $\mathcal{A}$ is also open, i.e., $\mathcal{A}=\operatorname{pint}(\mathcal{A})=\operatorname{int}(\mathcal{A})$. If $\mathcal{A}=\operatorname{pint}^{\diamond}(\mathcal{A})$, then $\mathcal{A}=\operatorname{pint}^{\diamond}(\mathcal{A})=\operatorname{int}^{\diamond}(\mathcal{A})$.

- If $\mathcal{A}=\operatorname{pint}^{(I)}(\mathcal{A})$, then $\mathcal{A}=\operatorname{pint}^{(I)}(\mathcal{A})=\operatorname{int}^{(I)}(\mathcal{A})$. If $\mathcal{A}=\operatorname{pint}^{(o l)}(\mathcal{A})$, then $\mathcal{A}=\operatorname{pint}^{(o l)}(\mathcal{A})=$ $\operatorname{int}^{(0 l)}(\mathcal{A})$.

- If $\mathcal{A}=\operatorname{pint}^{(I I)}(\mathcal{A})$, then $\mathcal{A}=\operatorname{pint}^{(I I)}(\mathcal{A})=\operatorname{int}^{(I I)}(\mathcal{A})$. If $\mathcal{A}=\operatorname{pint}^{(\text {oII })}(\mathcal{A})$, then $\mathcal{A}=\operatorname{pint}^{(\text {oII })}(\mathcal{A})=$ int $^{\text {(oll }}(\mathcal{A})$.

- If $\mathcal{A}=\operatorname{pint}^{(\text {III) }}(\mathcal{A})$, then $\mathcal{A}=\operatorname{pint}^{(\text {III) }}(\mathcal{A})=\operatorname{int}^{(\text {III) }}(\mathcal{A})$. If $\mathcal{A}=\operatorname{pint}{ }^{(\text {oIII) }}(\mathcal{A})$, then $\mathcal{A}=\operatorname{pint}^{(\text {oIII) }}(\mathcal{A})=$ int $^{(\text {(IIII) }}(\mathcal{A})$.

Proof. If $A$ is an pseudo-interior point, i.e., $A \in \operatorname{pint}(\mathcal{A})=\mathcal{A}$, then there exists $\epsilon>0$ such that $\mathcal{B}\left(A_{0} ; \epsilon\right) \subseteq \mathcal{A}$. As $A \in \mathcal{A}$, it follows that $A$ is also an interior point, i.e., $\operatorname{pint}(\mathcal{A}) \subseteq \operatorname{int}(\mathcal{A})$. From the first observation of Remark 5 , we obtain the desired result. The remaining cases can be similarly realized, and the proof is complete.

Proposition 11. Let $(\mathcal{I},\|\cdot\|)$ be a pseudo-seminormed interval space.

(i) Suppose that $\|\cdot\|$ satisfies the null superinequality.

- If $\mathcal{A}$ is any type of pseudo-open, then $A \in \mathcal{A}$ implies $A \oplus \omega \in \mathcal{A}$ for any $\omega \in \Omega$.

- If $\mathcal{A}$ is open, then $A \in \mathcal{A}$ implies $A \oplus \omega \in \operatorname{pint}(\mathcal{A})$ for any $\omega \in \Omega$.

- If $\mathcal{A}$ is type-I-open, then $A \in \mathcal{A}$ implies $A \oplus \omega \in \operatorname{pint}^{(I)}(\mathcal{A})$ for any $\omega \in \Omega$.

- If $\mathcal{A}$ is type-II-open, then $A \in \mathcal{A}$ implies $A \oplus \omega \in \operatorname{pint}^{(I I)}(\mathcal{A})$ for any $\omega \in \Omega$. 
- If $\mathcal{A}$ is type-III-open, then $A \in \mathcal{A}$ implies $A \oplus \omega \in \operatorname{pint}^{(I I I)}(\mathcal{A})$ for any $\omega \in \Omega$.

(ii) Suppose that $\|\cdot\|$ satisfies the null sub-inequality, and that $\mathcal{A}$ is any type of pseudo-open.

- $\quad A \oplus \omega \in \mathcal{A}$ implies $A \in \mathcal{A}$ for any $\omega \in \Omega$.

- $\mathcal{A} \oplus \omega \subseteq \mathcal{A}$ for any $\omega \in \Omega$ and $\mathcal{A} \oplus \Omega \subseteq \mathcal{A}$.

- $\quad A \oplus \omega \in \mathcal{A} \oplus \omega$ implies $A \in \mathcal{A}$ for any $\omega \in \Omega$.

- We have $\mathcal{A}=\mathcal{A} \oplus \Omega$.

(iii) Suppose that $\|\cdot\|$ satisfies the null sub-inequality, and that $\mathcal{A}$ is any type of $\diamond-p s e u d o-o p e n$. Then $A \in \mathcal{A}$ implies $A \oplus \omega \in \mathcal{A}$ for any $\omega \in \Omega$.

Proof. To prove part (i), suppose that $\mathcal{A}$ is type-III-pseudo-open set. For $A \in \mathcal{A}=\operatorname{pint}^{(\mathrm{III})}(\mathcal{A})$, by definition, there exists $\epsilon>0$ such that $\mathcal{B}(A ; \epsilon) \oplus \Omega \subseteq \mathcal{A} \oplus \Omega$. From part (i) of Proposition 7, we also have $\mathcal{B}(A \oplus \omega ; \epsilon) \oplus \Omega \subseteq \mathcal{A} \oplus \Omega$, which says that $A \oplus \omega \in \operatorname{pint}^{(I I I)}(\mathcal{A})=\mathcal{A}$. Now, we assume that $\mathcal{A}$ is type-III-open. Then $A \in \mathcal{A}=\operatorname{int}^{(\mathrm{III})}(\mathcal{A}) \subseteq \operatorname{pint}^{(\mathrm{III})}(\mathcal{A})$. We can also obtain $A \oplus \omega \in \operatorname{pint}^{(\mathrm{III}}(\mathcal{A})$. The other openness can be similarly obtained.

To prove the first case of part (ii), we consider the type-III-pseudo-open sets. If $A \oplus \omega \in \mathcal{A}=$ pint $^{(\text {III) }}(\mathcal{A})$, there exists $\epsilon>0$ such that $\mathcal{B}(A \oplus \omega ; \epsilon) \oplus \Omega \subseteq \mathcal{A} \oplus \Omega$. From part (ii) of Proposition 7, we also have $\mathcal{B}(A ; \epsilon) \oplus \Omega \subseteq \mathcal{A} \oplus \Omega$, which shows that $A \in \operatorname{pint}^{(\mathrm{III})}(\mathcal{A})=\mathcal{A}$.

To prove the second case of part (ii), we consider the type-III-pseudo-open sets. If $A \in \mathcal{A} \oplus \omega$, then $A=\widehat{A} \oplus \omega$ for some $\widehat{A} \in \mathcal{A}=\operatorname{pint}^{(\mathrm{III})}(\mathcal{A})$. Therefore there exists $\epsilon>0$ such that $\mathcal{B}(\widehat{A} ; \epsilon) \oplus \Omega \subseteq \mathcal{A} \oplus \Omega$. Since $\mathcal{B}(A ; \epsilon) \subseteq \mathcal{B}(A \oplus \omega ; \epsilon)=\mathcal{B}(\widehat{A} ; \epsilon)$ by part (ii) of Proposition 7, we see that $\mathcal{B}(A ; \epsilon) \oplus \Omega \subseteq \mathcal{A} \oplus \Omega$, i.e., $A \in$ pint $^{(I I I)}(\mathcal{A})=\mathcal{A}$. Now, for $A \in \mathcal{A} \oplus \Omega$, we see that $A \in \mathcal{A} \oplus \omega$ for some $\omega \in \Omega$, which implies $A \in \mathcal{A}$. Therefore we obtain $\mathcal{A} \oplus \Omega \subseteq \mathcal{A}$.

To prove the third case of part (ii), using the second case of part (ii), we have

$$
A \oplus \omega \in \mathcal{A} \oplus \omega \subseteq \mathcal{A} \oplus \Omega \subseteq \mathcal{A} .
$$

Using the first case of part (ii), we obtain $A \in \mathcal{A}$.

To prove the fourth case of part (ii), as $A=A \oplus[0,0]$ and $[0,0] \in \Omega$, it follows that $\mathcal{A} \subseteq \mathcal{A} \oplus \Omega$. By the second case of part (ii), we obtain the desired result.

To prove part (iii), from part (ii) of Proposition 7, we have $\mathcal{B}^{\diamond}(A \oplus \omega ; \epsilon) \subseteq \mathcal{B}^{\diamond}(A ; \epsilon)$. Therefore, using the similar argument in the proof of part (i), we can obtain the desired results. This completes the proof.

We remark that the results in Proposition 11 will not be true for any types of open sets. For example, in the proof of part (i), the inclusion $\mathcal{B}(A \oplus \omega ; \epsilon) \oplus \Omega \subseteq \mathcal{A} \oplus \Omega$ can just say that $A \oplus \omega \in \operatorname{pint}^{(I I I)}(\mathcal{A})$, as we do not know whether $A \oplus \omega$ is in $\mathcal{A}$ or not.

Proposition 12. Let $(\mathcal{I},\|\cdot\|)$ be a pseudo-seminormed interval space.

(i) Suppose that $\|\cdot\|$ satisfies the null condition.

- We have $\operatorname{int}(\mathcal{A})=\operatorname{int}^{(I)}(\mathcal{A}) \oplus \Omega \subseteq \mathcal{A}$. In particular, if $\mathcal{A}$ is open or type-I-open, then $\mathcal{A} \oplus \Omega \subseteq \mathcal{A}$.

- We have int ${ }^{(I I)}(\mathcal{A})=\operatorname{int}^{(I I I)}(\mathcal{A}) \subseteq \mathcal{A} \oplus \Omega$.

Moreover the concept of (resp. type-I, type-II, type-III) open set is equivalent to the concept of (resp. type-I, type-II, type-III) pseudo-open set.

(ii) Suppose that $\|\cdot\|$ satisfies the null sub-inequality. Then,

$$
\left(\operatorname{pint}^{(I I I)}(\mathcal{A})\right)^{c} \oplus \Omega=\left(\operatorname{pint}^{(I I)}(\mathcal{A})\right)^{c} \oplus \Omega \subseteq\left(\operatorname{pint}^{(I I)}(\mathcal{A})\right)^{c}=\left(\operatorname{pint}^{(I I I)}(\mathcal{A})\right)^{c} .
$$

In particular, if $\mathcal{A}$ is type-II-pseudo-open or type-III-pseudo-open, then $\mathcal{A}^{c} \oplus \Omega \subseteq \mathcal{A}^{c}$. 
Proof. To prove the first case of part (i), for any $A \in \operatorname{int}^{(\mathbb{I})}(\mathcal{A})$, there exists an open ball $\mathcal{B}(A ; \epsilon)$ such that $\mathcal{B}(A ; \epsilon) \oplus \Omega \subseteq \mathcal{A}$. As $A \in \mathcal{B}(A ; \epsilon)$, by the first observation of Remark 2, we have $A \oplus \Omega \subseteq$ $\mathcal{B}(A ; \epsilon) \oplus \Omega \subseteq \mathcal{A}$. This shows int ${ }^{(\mathrm{II}}(\mathcal{A}) \oplus \Omega \subseteq \mathcal{A}$. Using Remark 6, we obtain the desired results.

To prove the second case of part (i), for any $A \in \operatorname{int}^{(I I)}(\mathcal{A})$, there exists an open ball $\mathcal{B}(A ; \epsilon)$ such that $\mathcal{B}(A ; \epsilon) \subseteq \mathcal{A} \oplus \Omega$. Then we have $A \in \mathcal{A} \oplus \Omega$, since $A \in \mathcal{B}(A ; \epsilon)$. This shows int ${ }^{(I I)}(\mathcal{A}) \subseteq \mathcal{A} \oplus \Omega$. Using Remark 6, we obtain the desired results. From Remark 5, we see that the concept of (resp. type-I, type-II, type-III) open set is equivalent to the concept of (resp. type-I, type-II, type-III) pseudo-open set.

To prove part (ii), for any $A \in\left(\operatorname{pint}^{(\mathrm{II})}(\mathcal{A})\right)^{c} \oplus \Omega$, we have $A=\widehat{A} \oplus \widehat{\omega}$ for some $\widehat{A} \in\left(\operatorname{pint}^{(\mathrm{II})}(\mathcal{A})\right)^{c}$ and $\widehat{\omega} \in \Omega$. By definition, we see that $\mathcal{B}(\widehat{A} ; \epsilon) \nsubseteq \mathcal{A} \oplus \Omega$ for every $\epsilon>0$. By part (ii) of Proposition 7, we also have $\mathcal{B}(A ; \epsilon) \nsubseteq \mathcal{A} \oplus \Omega$ for every $\epsilon>0$. This says that $A$ is not an type-II-pseudo-interior point of $\mathcal{A}$, i.e., $A \notin \operatorname{pint}^{(\mathrm{II})}(\mathcal{A})$. This completes the proof.

Proposition 13. Let $(\mathcal{I},\|\cdot\|)$ be a pseudo-seminormed interval space.

(i) $\mathcal{B}^{\diamond}\left(A_{0} ; \epsilon\right)$ is $\diamond$-open, $\diamond$-type-II-open, and $\diamond$-type-III-open. We also have the inclusions $\mathcal{B}^{\diamond}\left(A_{0} ; \epsilon\right) \subseteq$ $\operatorname{pint}\left(\mathcal{B}^{\diamond}\left(A_{0} ; \epsilon\right)\right), \mathcal{B}^{\diamond}\left(A_{0} ; \epsilon\right) \subseteq \operatorname{pint}^{(\text {oIII }}\left(\mathcal{B}^{\diamond}\left(A_{0} ; \epsilon\right)\right)$, and $\mathcal{B}^{\diamond}\left(A_{0} ; \epsilon\right) \subseteq \operatorname{pint}^{\text {(oIII) }}\left(\mathcal{B}^{\diamond}\left(A_{0} ; \epsilon\right)\right)$.

(ii) $\mathcal{B}\left(A_{0} ; \epsilon\right)$ is open, type-II-open, and type-III-open. We also have the inclusions $\mathcal{B}\left(A_{0} ; \epsilon\right) \subseteq$ $\operatorname{pint}\left(\mathcal{B}\left(A_{0} ; \epsilon\right)\right), \mathcal{B}\left(A_{0} ; \epsilon\right) \subseteq \operatorname{pint}^{(I I)}\left(\mathcal{B}\left(A_{0} ; \epsilon\right)\right)$, and $\mathcal{B}\left(A_{0} ; \epsilon\right) \subseteq \operatorname{pint}^{(I I I)}\left(\mathcal{B}\left(A_{0} ; \epsilon\right)\right)$.

(iii) Suppose that $\|\cdot\|$ satisfies the null sub-inequality. Then $\mathcal{B}^{\diamond}\left(A_{0} ; \epsilon\right)$ is $\diamond$-type-I-open and $\mathcal{B}\left(A_{0} ; \epsilon\right)$ is type-I-open. We also have the inclusions $\mathcal{B}^{\diamond}\left(A_{0} ; \epsilon\right) \subseteq \operatorname{pint}^{(\mathrm{oll})}\left(\mathcal{B}^{\diamond}\left(A_{0} ; \epsilon\right)\right)$, and $\mathcal{B}\left(A_{0} ; \epsilon\right) \subseteq$ $\operatorname{pint}^{(I)}\left(\mathcal{B}\left(A_{0} ; \epsilon\right)\right)$.

Proof. To prove part (i), for any $A \in \mathcal{B}^{\diamond}\left(A_{0} ; \epsilon\right)$, we have $A=A_{0} \oplus C$ with $\|C\|<\epsilon$. Let $\widehat{\epsilon}=\epsilon-\|$ $C \|>0$. For any $\widehat{A} \in \mathcal{B}^{\diamond}(A ; \widehat{\epsilon})$, i.e., $\widehat{A}=A \oplus D$ with $\|D\|<\widehat{\epsilon}$, we obtain $\widehat{A}=A_{0} \oplus C \oplus D$ and

$$
\|C \oplus D\| \leq\|C\|+\|D\|=\epsilon-\widehat{\epsilon}+\|D\|<\epsilon-\widehat{\epsilon}+\widehat{\epsilon}=\epsilon,
$$

which means that $\widehat{A} \in \mathcal{B}^{\diamond}\left(A_{0} ; \epsilon\right)$, i.e.,

$$
\mathcal{B}^{\diamond}(A ; \widehat{\epsilon}) \subseteq \mathcal{B}^{\diamond}\left(A_{0} ; \epsilon\right) .
$$

This shows that $\mathcal{B}^{\diamond}\left(A_{0} ; \epsilon\right) \subseteq \operatorname{int}\left(\mathcal{B}^{\diamond}\left(A_{0} ; \epsilon\right)\right)$. Therefore, we obtain $\mathcal{B}^{\diamond}\left(A_{0} ; \epsilon\right)=\operatorname{int}\left(\mathcal{B}^{\diamond}\left(A_{0} ; \epsilon\right)\right)$. We can similarly obtain the inclusion $\mathcal{B}^{\diamond}\left(A_{0} ; \epsilon\right) \subseteq \operatorname{pint}\left(\mathcal{B}^{\diamond}\left(A_{0} ; \epsilon\right)\right)$. However, we cannot have the equality $\mathcal{B}^{\diamond}\left(A_{0} ; \epsilon\right)=\operatorname{pint}\left(\mathcal{B}^{\diamond}\left(A_{0} ; \epsilon\right)\right)$, as $\operatorname{pint}\left(\mathcal{B}^{\diamond}\left(A_{0} ; \epsilon\right)\right)$ is not necessarily contained in $\mathcal{B}^{\diamond}\left(A_{0} ; \epsilon\right)$. From (6), we have $\mathcal{B}^{\diamond}(x ; \widehat{\epsilon}) \oplus \Omega \subseteq \mathcal{B}^{\diamond}\left(A_{0} ; \epsilon\right) \oplus \Omega$. This says that $\mathcal{B}^{\diamond}\left(A_{0} ; \epsilon\right)$ is $\diamond$-type-III-open. On the other hand, from (6) and part (ii) of Proposition 9, we also have

$$
\mathcal{B}^{\diamond}(A ; \widehat{\epsilon}) \subseteq \mathcal{B}^{\diamond}\left(A_{0} ; \epsilon\right) \subseteq \mathcal{B}^{\diamond}\left(A_{0} ; \epsilon\right) \oplus \Omega
$$

This shows that $\mathcal{B}^{\diamond}\left(A_{0} ; \epsilon\right)$ is $\diamond$-type-II-open.

To prove part (ii), for any $A \in \mathcal{B}\left(A_{0} ; \epsilon\right)$, we have $\left\|A \ominus A_{0}\right\|<\epsilon$. Let $\widehat{\epsilon}=\left\|A \ominus A_{0}\right\|$. For any $\widehat{A} \in \mathcal{B}(A ; \epsilon-\widehat{\epsilon})$, we have $\|\widehat{A} \ominus A\|<\epsilon-\widehat{\epsilon}$. Therefore, by Proposition 5 , we obtain

$$
\left\|\widehat{A} \ominus A_{0}\right\| \leq\|\widehat{A} \ominus A\|+\left\|A \ominus A_{0}\right\|=\widehat{\epsilon}+\|\widehat{A} \ominus A\|<\widehat{\epsilon}+\epsilon-\widehat{\epsilon}=\epsilon,
$$

which means that $\widehat{A} \in \mathcal{B}\left(A_{0} ; \epsilon\right)$, i.e.,

$$
\mathcal{B}(A ; \epsilon-\widehat{\epsilon}) \subseteq \mathcal{B}\left(A_{0} ; \epsilon\right) .
$$

This shows that $\mathcal{B}\left(A_{0} ; \epsilon\right) \subseteq \operatorname{int}\left(\mathcal{B}\left(A_{0} ; \epsilon\right)\right)$. Therefore, we obtain $\mathcal{B}\left(A_{0} ; \epsilon\right)=\operatorname{int}\left(\mathcal{B}\left(A_{0} ; \epsilon\right)\right)$. We can similarly obtain the inclusion $\mathcal{B}\left(A_{0} ; \epsilon\right) \subseteq \operatorname{pint}\left(\mathcal{B}\left(A_{0} ; \epsilon\right)\right)$. From $(7)$, we have $\mathcal{B}(A ; \epsilon-\widehat{\epsilon}) \oplus \Omega \subseteq$ $\mathcal{B}\left(A_{0} ; \epsilon\right) \oplus \Omega$. 
This says that $\mathcal{B}\left(A_{0} ; \epsilon\right)$ is type-III-open. On the other hand, from (7) and part (ii) of Proposition 9, we also have

$$
\mathcal{B}(A ; \epsilon-\widehat{\epsilon}) \subseteq \mathcal{B}\left(A_{0} ; \epsilon\right) \subseteq \mathcal{B}\left(A_{0} ; \epsilon\right) \oplus \Omega .
$$

This shows that $\mathcal{B}\left(A_{0} ; \epsilon\right)$ is type-II-open.

To prove part (iii), from (6), (7), and part (ii) of Proposition 9, we have

$$
\mathcal{B}^{\diamond}(A ; \widehat{\epsilon}) \oplus \Omega \subseteq \mathcal{B}^{\diamond}\left(A_{0} ; \epsilon\right) \oplus \Omega=\mathcal{B}^{\diamond}\left(A_{0} ; \epsilon\right)
$$

and

$$
\mathcal{B}(A ; \epsilon-\widehat{\epsilon}) \oplus \Omega \subseteq \mathcal{B}\left(A_{0} ; \epsilon\right) \oplus \Omega=\mathcal{B}\left(A_{0} ; \epsilon\right) .
$$

This shows that $\mathcal{B}^{\diamond}\left(A_{0} ; \epsilon\right)$ is $\diamond$-type-I-open, and that $\mathcal{B}\left(A_{0} ; \epsilon\right)$ is type-I-open. We complete the proof.

Proposition 14. Let $(\mathcal{I},\|\cdot\|)$ be a pseudo-seminormed interval space. Suppose that the center $A_{0}$ is in the open balls $\mathcal{B}\left(A_{0} ; \epsilon\right)$ and $\mathcal{B}^{\diamond}\left(A_{0} ; \epsilon\right)$. The following statements hold true.

(i) $\mathcal{B}\left(A_{0} ; \epsilon\right)$ is pseudo-open and $\diamond$-pseudo-open.

(ii) Suppose that $\|$. \| satisfies the null sub-inequality. Then, $\mathcal{B}\left(A_{0} ; \epsilon\right)$ is type-I-pseudo-open, type-II-pseudo-open and type-III-pseudo-open.

(iii) Suppose that $\|\cdot\|$ satisfies the null sub-inequality. Then, $\mathcal{B}\left(A_{0} ; \epsilon\right)$ is $\diamond$-type-I-pseudo-open, $\diamond$-type-II-pseudo-open and $\diamond$-type-III-pseudo-open.

Proof. The results follow from Proposition 13, Remark 8 and part (ii) of Proposition 9 immediately.

\section{Topoloigcal Spaces}

Now, we are in a position to investigate the topological structure generated by the pseudo-seminormed interval space $(\mathcal{I},\|\cdot\|)$ based on the different kinds of openness. We denote by $\tau_{0}$ and $\tau_{0}^{(\diamond)}$ the set of all open and $\diamond$-open subsets of $\mathcal{I}$, respectively, and by $\mathrm{p} \tau_{0}$ and $\mathrm{p} \tau_{0}^{(\diamond)}$ the set of all pseudo-open and $\diamond$-pseudo-open subsets of $\mathcal{I}$, respectively. We denote by $\tau^{(\mathrm{l})}$ and $\tau^{(\mathrm{(l)})}$ the set of all type-I-open and $\diamond$-type-I-open subsets of $\mathcal{I}$, respectively, and by $\mathrm{p} \tau^{(\mathrm{I})}$ and $\mathrm{p} \tau^{(\mathrm{ol})}$ the set of all type-I-pseudo-open and $\diamond$-type-I-pseudo-open subsets of $\mathcal{I}$, respectively. We can similarly define the families $\tau^{(\mathrm{II})}, \tau^{(\mathrm{III})}, \tau^{(\mathrm{oII})}, \tau^{(\mathrm{oIII})}, \mathrm{p} \tau^{(\mathrm{III})}, \mathrm{p} \tau^{(\mathrm{III})}, \mathrm{p} \tau^{(\mathrm{oII})}$ and $\mathrm{p} \tau^{(\mathrm{oIII})}$.

Proposition 15. Let $(\mathcal{I},\|\cdot\|)$ be a pseudo-seminormed interval space.

(i) $\left(\mathcal{I}, \tau^{(I)}\right)$ and $\left(\mathcal{I}, \tau^{(\text {oll })}\right)$ are topological spaces.

(ii) Suppose that each open ball $\mathcal{B}\left(A_{0} ; \epsilon\right)$ contains the center $A_{0}$. Then $\left(\mathcal{I}, p \tau^{(I)}\right)=\left(\mathcal{I}, \tau^{(I)}\right)$ is a topological space.

(iii) Suppose that each open ball $\mathcal{B}^{\diamond}\left(A_{0} ; \epsilon\right)$ contains the center $A_{0}$. Then $\left(\mathcal{I}, p \tau^{(o D)}\right)=\left(\mathcal{I}, \tau^{(o l)}\right)$ is a topological space.

Proof. To prove part (i), by the second observation of Remark 9, we see that $\varnothing \in \tau^{(\mathrm{I})}$ and $X \in \tau^{(\mathrm{I})}$. Let $\mathcal{A}=\bigcap_{i=1}^{n} \mathcal{A}_{i}$, where $\mathcal{A}_{i}$ are type-I-open sets for all $i=1, \cdots, n$. For $A \in \mathcal{A}$, we have $A \in \mathcal{A}_{i}$ for all $i=1, \cdots, n$. Then, there exists $\epsilon_{i}$ such that $\mathcal{B}\left(A ; \epsilon_{i}\right) \oplus \Omega \subseteq \mathcal{A}_{i}$ for all $i=1, \cdots, n$. Let $\epsilon=\min \left\{\epsilon_{1}, \cdots, \epsilon_{n}\right\}$. Then $\mathcal{B}(A ; \epsilon) \oplus \Omega \subseteq \mathcal{B}\left(A ; \epsilon_{i}\right) \oplus \Omega \subseteq \mathcal{A}_{i}$ for all $i=1, \cdots, n$, which says that $\mathcal{B}(A ; \epsilon) \oplus \Omega \subseteq \bigcap_{i=1}^{n} \mathcal{A}_{i}=\mathcal{A}$, i.e., $\mathcal{A} \subseteq \operatorname{int}^{(\mathrm{I})}(\mathcal{A})$. Therefore the intersection $\mathcal{A}$ is type-I-open by Remark 5. On the other hand, let $\mathcal{A}=\bigcup_{\delta} \mathcal{A}_{\delta}$. Then, $A \in \mathcal{A}$ implies that $A \in \mathcal{A}_{\delta}$ for some $\delta$. This says that $\mathcal{B}(A ; \epsilon) \oplus \Omega \subseteq \mathcal{A}_{\delta} \subseteq \mathcal{A}$ for some $\epsilon>0$, i.e., $\mathcal{A} \subseteq \operatorname{int}^{(\mathbb{I})}(\mathcal{A})$. Therefore, the union $\mathcal{A}$ is type-I-open. This shows that $\left(\mathcal{I}, \tau^{(\mathrm{I})}\right)$ is a topological space. For the case of $\diamond$-type-I-open subsets of $\mathcal{I}$, we can similarly obtain the desired result. Parts (ii) and (iii) follow from Remark 8 and part (i) immediately. This completes the proof. 
We remark that Remark 2 shows the sufficient conditions for the open balls $\mathcal{B}(A ; \epsilon)$ and $\mathcal{B}^{\diamond}(A ; \epsilon)$ containing the center $A$.

Proposition 16. Let $(\mathcal{I},\|\cdot\|)$ be a pseudo-seminormed interval space.

(i) $\left(\mathcal{I}, \tau_{0}\right)$ and $\left(\mathcal{I}, \tau_{0}^{(\diamond)}\right)$ are topological spaces.

(ii) Suppose that each open ball $\mathcal{B}\left(A_{0} ; \epsilon\right)$ contains the center $A_{0}$. Then, $\left(\mathcal{I}, \tau_{0}\right)=\left(\mathcal{I}, p \tau_{0}\right)$ is a topological space.

(iii) Suppose that each open ball $\mathcal{B}^{\diamond}\left(A_{0} ; \epsilon\right)$ contains the center $A_{0}$. Then, $\left(\mathcal{I}, \tau_{0}^{(\diamond)}\right)=\left(\mathcal{I}, p \tau_{0}^{(\diamond)}\right)$ is a topological space.

Proof. The empty set $\varnothing$ and $\mathcal{I}$ are open by the first observation of Remark 9 . The remaining proof follows from the similar argument of Proposition 15 without considering the null set $\Omega$.

Let $(\mathcal{I},\|\cdot\|)$ be a pseudo-seminormed interval space. We consider the following families,

$$
\widetilde{\tau}^{(\mathrm{II})}=\left\{\mathcal{A} \in \tau^{(\mathrm{II})}: \mathcal{A} \oplus \Omega \subseteq \mathcal{A}\right\}
$$

and

$$
\widetilde{\tau}^{(I I I)}=\left\{\mathcal{A} \in \tau^{(\mathrm{IIII})}: \mathcal{A} \oplus \Omega \subseteq \mathcal{A}\right\} .
$$

We can similarly define $\widetilde{\tau}^{(\diamond \mathrm{II})}$ and $\widetilde{\tau}^{(\odot \mathrm{III})}$. Then, $\widetilde{\tau}^{(\mathrm{II})} \subseteq \tau^{(\mathrm{II})}, \widetilde{\tau}^{(\mathrm{III})} \subseteq \tau^{(\mathrm{III})}, \widetilde{\tau}^{(\diamond \mathrm{II})} \subseteq \tau^{(\diamond \mathrm{III})}$, and $\widetilde{\tau}^{(\diamond \mathrm{III})} \subseteq \tau^{(\diamond \mathrm{III})}$. We can also similarly define $\widetilde{\mathrm{p}}^{(\mathrm{II})}, \widetilde{\mathrm{p}}^{(\mathrm{III})}, \widetilde{\mathrm{p}}^{(\odot \mathrm{II})}$, and $\widetilde{\mathrm{p}}^{(\odot \mathrm{III})}$ regarding the pseudo-openness. Then, $\widetilde{\mathrm{p}} \tau^{(\mathrm{II})} \subseteq \mathrm{p} \tau^{(\mathrm{II})}, \widetilde{\mathrm{p}} \tau^{(\mathrm{III})} \subseteq \mathrm{p} \tau^{(\mathrm{III})}, \widetilde{\mathrm{p}} \tau^{(\mathrm{oII})} \subseteq \mathrm{p} \tau^{(\mathrm{SII})}$, and $\widetilde{\mathrm{p}} \tau^{(\text {(III) }} \subseteq \mathrm{p} \tau^{(\odot \mathrm{III})}$. Suppose that $\|\cdot\|$ satisfies the null sub-inequality.

Proposition 17. Let $(\mathcal{I},\|\cdot\|)$ be a pseudo-seminormed interval space. Suppose that $\|\cdot\|$ satisfies the null sub-inequality. Then,

$$
\widetilde{\mathrm{p}}^{(I I)}=\mathrm{p} \tau^{(I I)}=\mathrm{p} \tau^{(I I I)}=\widetilde{\mathrm{p} \tau} \widetilde{(I I I)}^{(\text {nd }} \widetilde{\tau}^{(I I)}=\tau^{(I I)}=\tau^{(I I I)}=\widetilde{\tau}^{(I I I)} .
$$

Proof. The results follow from Remark 6 and part (ii) of Proposition 11 immediately.

Proposition 18. Let $(\mathcal{I},\|\cdot\|)$ be a pseudo-seminormed interval space.

(i) $\left(\mathcal{I}, \widetilde{\tau}^{(I I)}\right)$ and $\left(\mathcal{I}, \widetilde{\tau}^{(\circ I I)}\right)$ are topological spaces.

(ii) The following statements hold true.

- $\quad$ Suppose that each open ball $\mathcal{B}(A ; \epsilon)$ contains the center $A$. Then, $\left(\mathcal{I}, \widetilde{p \tau}^{(I I)}\right)=\left(\mathcal{I}, \widetilde{\tau}^{(I I)}\right)$ is a topological space.

- $\quad$ Suppose that each open ball $\mathcal{B}^{\diamond}(A ; \epsilon)$ contains the center $A$. Then, $\left(\mathcal{I}, \widetilde{p \tau}^{(\diamond I I)}\right)=\left(\mathcal{I}, \widetilde{\tau}^{(\diamond I I)}\right)$ is a topological space.

Proof. To prove part (i), given $\mathcal{A}_{1}, \mathcal{A}_{2} \in \widetilde{\tau}^{(\mathrm{II})}$, let $\mathcal{A}=\mathcal{A}_{1} \cap \mathcal{A}_{2}$. For $A \in \mathcal{A}$, we have $A \in \mathcal{A}_{i}$ for $i=1,2$. Then, there exist $\epsilon_{i}$ such that $\mathcal{B}\left(A ; \epsilon_{i}\right) \subseteq \mathcal{A}_{i} \oplus \Omega$ for all $i=1,2$. Let $\epsilon=\min \left\{\epsilon_{1}, \epsilon_{2}\right\}$. Then

$$
\mathcal{B}(A ; \epsilon) \subseteq \mathcal{B}\left(A ; \epsilon_{i}\right) \subseteq \mathcal{A}_{i} \oplus \Omega
$$

for all $i=1,2$, which says that

$$
\mathcal{B}(A ; \epsilon) \subseteq\left[\left(\mathcal{A}_{1} \oplus \Omega\right) \cap\left(\mathcal{A}_{2} \oplus \Omega\right)\right]=\left(\mathcal{A}_{1} \cap \mathcal{A}_{2}\right) \oplus \Omega=\mathcal{A} \oplus \Omega
$$

by Proposition 4 . This shows that $\mathcal{A}$ is type-II-open. For $A \in \mathcal{A} \oplus \Omega$, we have $A=\bar{A} \oplus \omega$ for some $\bar{A} \in \mathcal{A}$ and $\omega \in \Omega$. Since $\bar{A} \in \mathcal{A}_{1} \cap \mathcal{A}_{2}$, it follows that $A \in \mathcal{A}_{1} \oplus \Omega \subseteq \mathcal{A}_{1}$ and $A \in \mathcal{A}_{2} \oplus \Omega \subseteq \mathcal{A}_{2}$, 
which says that $A \in \mathcal{A}_{1} \cap \mathcal{A}_{2}=\mathcal{A}$, i.e., $\mathcal{A} \oplus \Omega \subseteq \mathcal{A}$. This shows that $\mathcal{A}$ is indeed in $\widetilde{\tau}^{(\mathrm{II})}$. Therefore, the intersection of finitely many members of $\widetilde{\tau}^{(\mathrm{II})}$ is a member of $\widetilde{\tau}^{(\mathrm{II})}$.

Now, given a family $\left\{\mathcal{A}_{\delta}\right\}_{\delta \in \Lambda} \subset \tau^{(\text {II) }}$, let $\mathcal{A}=\bigcup_{\delta \in \Lambda} \mathcal{A}_{\delta}$. Then, $A \in \mathcal{A}$ implies that $A \in \mathcal{A}_{\delta}$ for some $\delta \in \Lambda$. This says that

$$
\mathcal{B}(A ; \epsilon) \subseteq \mathcal{A}_{\delta} \oplus \Omega \subseteq \mathcal{A} \oplus \Omega
$$

for some $\epsilon>0$. Therefore, the union $A$ is type-II-open. For $A \in \mathcal{A} \oplus \Omega$, we have $A=\bar{A} \oplus \omega$, where $\bar{A} \in \mathcal{A}$, i.e., $\bar{A} \in \mathcal{A}_{\delta}$ for some $\delta \in \Lambda$. It also says that $A \in \mathcal{A}_{\delta} \oplus \Omega \subseteq \mathcal{A}_{\delta} \subseteq \mathcal{A}$, i.e., $\mathcal{A} \oplus \Omega \subseteq \mathcal{A}$. This shows that $\mathcal{A}$ is indeed in $\widetilde{\tau}^{\text {(II) }}$. By the third observation of Remark 9, we see that $\varnothing$ and $\mathcal{I}$ are also type-II-open. It is obvious that $\varnothing \oplus \Omega=\varnothing$ and $\mathcal{I} \oplus \Omega \subseteq \mathcal{I}$, which shows that $\varnothing, X \in \widetilde{\tau}^{(\mathrm{II})}$. Therefore, $\left(\mathcal{I}, \widetilde{\tau}^{(\mathrm{II})}\right)$ is indeed a topological space. The above arguments are also valid for $\widetilde{\tau}^{(\mathrm{IIII})}$.

Part (ii) follows immediately from the third observation of Remark 8 and part (i). This completes the proof.

Proposition 19. Let $(\mathcal{I},\|\cdot\|)$ be a pseudo-seminormed interval space.

(i) $\left(\mathcal{I}, \widetilde{\tau}^{(I I I)}\right)$ and $\left(\mathcal{I}, \widetilde{\tau}^{(\text {oIII) })}\right.$ are topological spaces.

(ii) The following statements hold true.

- $\quad$ Suppose that each open ball $\mathcal{B}(A ; \epsilon)$ contains the center $A$. Then $\left(\mathcal{I}, \widetilde{p \tau}^{(I I I)}\right)=\left(\mathcal{I}, \widetilde{\tau}^{(I I I)}\right)$ is a topological space.

- $\quad$ Suppose that each open ball $\mathcal{B}^{\diamond}(A ; \epsilon)$ contains the center $A$. Then $\left(\mathcal{I}, \widetilde{p \tau}^{(\text {oIII) }}\right)=\left(\mathcal{I}, \widetilde{\tau}^{(\text {(oIII) }}\right)$ is a topological space.

Proof. To prove part (i), by the fourth observation of Remark 9, it is clear to see that $\varnothing, \mathcal{I} \in \tau^{(\mathrm{IIII})}$. As $\varnothing \oplus \Omega=\varnothing$ and $\mathcal{I} \oplus \Omega \subseteq \mathcal{I}$, it follows that $\varnothing, \mathcal{I} \in \widetilde{\tau}^{\text {(III) }}$. Given $\mathcal{A}_{1}, \mathcal{A}_{2} \in \widetilde{\tau}^{\text {(III) }}$, let $\mathcal{A}=\mathcal{A}_{1} \cap \mathcal{A}_{2}$. For $A \in \mathcal{A}$, there exist $\epsilon_{i}$ such that $\mathcal{B}\left(A ; \epsilon_{i}\right) \oplus \Omega \subseteq \mathcal{A}_{i} \oplus \Omega$ for all $i=1,2$. Let $\epsilon=\min \left\{\epsilon_{1}, \epsilon_{2}\right\}$. Then,

$$
\mathcal{B}(A ; \epsilon) \oplus \Omega \subseteq \mathcal{B}\left(A ; \epsilon_{i}\right) \oplus \Omega \subseteq \mathcal{A}_{i} \oplus \Omega
$$

for all $i=1,2$, which says that

$$
\mathcal{B}(A ; \epsilon) \oplus \Omega \subseteq\left[\left(\mathcal{A}_{1} \oplus \Omega\right) \cap\left(\mathcal{A}_{2} \oplus \Omega\right)\right]=\left(\mathcal{A}_{1} \cap \mathcal{A}_{2}\right) \oplus \Omega=\mathcal{A} \oplus \Omega
$$

by Proposition 4 . This shows that $\mathcal{A}$ is type-III-open. From the proof of Proposition 18, we also see that $\mathcal{A} \oplus \Omega \subseteq \mathcal{A}$. Therefore, the intersection of finitely many members of $\widetilde{\tau}^{(\text {III) }}$ is a member of $\widetilde{\tau}^{(\text {III) }}$.

Now, given a family $\left\{\mathcal{A}_{\delta}\right\} \subset \widetilde{\tau}^{(I I I)}$, let $\mathcal{A}=\bigcup_{\delta} \mathcal{A}_{\delta}$. Then, $A \in \mathcal{A}$ implies that $A \in \mathcal{A}_{\delta}$ for some $\delta$. This says that

$$
\mathcal{B}(A ; \epsilon) \oplus \Omega \subseteq \mathcal{A}_{\delta} \oplus \Omega \subseteq \mathcal{A} \oplus \Omega
$$

for some $\epsilon>0$. Therefore, the union $\mathcal{A}$ is type-III-open. From the proof of Proposition 18, we also see that $\mathcal{A} \oplus \Omega \subseteq \mathcal{A}$, i.e., $\mathcal{A} \in \widetilde{\tau}^{(\mathrm{III})}$. This shows that $\left(\mathcal{I}, \widetilde{\tau}^{(\mathrm{III})}\right)$ is indeed a topological space. The above arguments are also valid for $\widetilde{\tau}^{(\text {(III) }}$.

Part (ii) follows immediately from the fourth observation of Remark 8 and part (i). This completes the proof.

Proposition 20. Let $(\mathcal{I},\|\cdot\|)$ be a pseudo-seminormed interval space. Suppose that $\|\cdot\|$ satisfies the null sub-inequality. If each open ball $\mathcal{B}(A ; \epsilon)$ contains the center $A$, then $\left(\mathcal{I}, \mathrm{p} \tau^{(I I)}\right)=\left(\mathcal{I}, \mathrm{p} \tau^{(I I I)}\right)$ is a topological space.

Proof. By the third observation of Remark 9, we see that $\varnothing, \mathcal{I} \in \mathrm{p} \tau^{\text {(II). }}$ Given $\mathcal{A}_{1}, \mathcal{A}_{2} \in \mathrm{p} \tau^{(\mathrm{II})}$, let $\mathcal{A}=\mathcal{A}_{1} \cap \mathcal{A}_{2}$. We want to show $\mathcal{A}=\operatorname{pint}^{(\mathrm{II})}(\mathcal{A})$. For $A \in \mathcal{A}$, we have $A \in \mathcal{A}_{i}$ for $i=1$, 2. There exist $\epsilon_{i}$ 
such that $\mathcal{B}\left(A ; \epsilon_{i}\right) \subseteq \mathcal{A}_{i} \oplus \Omega$ for all $i=1$, 2. Let $\epsilon=\min \left\{\epsilon_{1}, \epsilon_{2}\right\}$. Then $\mathcal{B}(A ; \epsilon) \subseteq \mathcal{B}\left(A ; \epsilon_{i}\right) \subseteq \mathcal{A}_{i} \oplus \Omega$ for $i=1,2$, which says that, using part (ii) of Proposition 11,

$$
\mathcal{B}(A ; \epsilon) \subseteq\left[\left(\mathcal{A}_{1} \oplus \Omega\right) \cap\left(\mathcal{A}_{2} \oplus \Omega\right)\right]=\mathcal{A}_{1} \cap \mathcal{A}_{2}=\left(\mathcal{A}_{1} \cap \mathcal{A}_{2}\right) \oplus \Omega=\mathcal{A} \oplus \Omega .
$$

This shows that $A \in \operatorname{int}^{(\text {II) }}(\mathcal{A})$, i.e., $\mathcal{A} \subseteq \operatorname{int}^{(\mathrm{II})}(\mathcal{A}) \subseteq \operatorname{pint}^{(\mathrm{II})}(\mathcal{A})$ by Remark 5. On the other hand, for $A \in \operatorname{pint}^{(\mathrm{II}}(\mathcal{A})$, using part (ii) of Proposition 11, we have

$$
A \in \mathcal{B}(A ; \epsilon) \subseteq \mathcal{A} \oplus \Omega=\left(\mathcal{A}_{1} \cap \mathcal{A}_{2}\right) \oplus \Omega \subseteq \mathcal{A}_{1} \oplus \Omega=\mathcal{A}_{1} .
$$

We can similarly obtain $A \in \mathcal{A}_{2}$, i.e., $A \in \mathcal{A}_{1} \cap \mathcal{A}_{2}=\mathcal{A}$. This shows that pint ${ }^{(\mathrm{II})}(\mathcal{A}) \subseteq \mathcal{A}$. Therefore, we conclude that the intersection of finitely many members of $\mathrm{p} \tau^{(\mathrm{II})}$ is a member of $\mathrm{p} \tau^{(\mathrm{II})}$.

Now, given a family $\left\{\mathcal{A}_{\delta}\right\}_{\delta \in \Lambda} \subset \mathrm{p} \tau^{(\mathrm{II})}$, let $\mathcal{A}=\bigcup_{\delta \in \Lambda} \mathcal{A}_{\delta}$. Then $A \in \mathcal{A}$ implies that $A \in \mathcal{A}_{\delta}$ for some $\delta \in \Lambda$. This says that

$$
\mathcal{B}(A ; \epsilon) \subseteq \mathcal{A}_{\delta} \oplus \Omega \subseteq \mathcal{A} \oplus \Omega
$$

for some $\epsilon>0$. Therefore, we obtain $\mathcal{A} \subseteq \operatorname{int}^{(I I)}(\mathcal{A}) \subseteq \operatorname{pint}^{(I I)}(\mathcal{A})$. On the other hand, for $A \in \operatorname{pint}^{(I I)}(\mathcal{A})$, we have

$$
A \in \mathcal{B}(A ; \epsilon) \subseteq \mathcal{A} \oplus \Omega=\mathcal{A}
$$

by part (ii) of Proposition 11. This shows that $\operatorname{pint}^{(I I)}(\mathcal{A}) \subseteq \mathcal{A}$, i.e., $\mathcal{A}=\operatorname{pint}^{(I I)}(\mathcal{A})$. Therefore, by Remark 6, we conclude that $\left(\mathcal{I}, \mathrm{p} \tau^{(\mathrm{II})}\right)=\left(\mathcal{I}, \mathrm{p} \tau^{(\mathrm{III})}\right)$ is a topological space. This completes the proof.

Funding: This research received no external funding.

Acknowledgments: The author would like to thank the reviewers for suggesting the useful comments.

Conflicts of Interest: The author declares no conflicts of interest.

\section{References}

1. Alefeld, G.; Herzberger, J. Introduction to Interval Computations; Academic Press: New York, NY, USA, 1983.

2. Eijgenraam, P. The Solution of Initial Value Problems Using Interval Arithmetic; Mathematisch Centrum: Amsterdam, The Netherlands, 1981.

3. Moore, R.E. Interval Analysis; Prentice-Hall: Englewood Cliffs, NJ, USA, 1966.

4. Moore, R.E. Method and Applications of Interval Analysis; SIAM: Philadelphia, PA, USA, 1979.

5. Neumaier, A. Interval Methods for Systems of Equations; Cambridge University Press: New York, NY, USA, 1990.

6. Wu, H.-C. The Karush-Kuhn-Tucker Optimality Conditions in Multiobjective Programming Problem with Multiple Interval-Valued Objective Functions. Eur. J. Oper. Res. 2009, 196, 49-60 [CrossRef]

7. Wu, H.-C. Duality Theory in Interval-Valued Linear Programming Problems. J. Optim. Theory Appl. 2011, 150, 298-316. [CrossRef]

8. $\mathrm{Wu}, \mathrm{H}$.-C. Interval-Valued Cores and Interval-Valued Dominance Cores of Cooperative Games Endowed with Interval-Valued Payoffs. Mathematics 2018, 6, 255. [CrossRef]

9. $\mathrm{Wu}, \mathrm{H} .-\mathrm{C}$. Solving the Interval-Valued Optimization Problems Based on the Concept of Null Set. J. Ind. Manag. Optim. 2018, 14, 1157-1178. [CrossRef]

10. Wu, H.-C. Hahn-Banach Theorems in Normed Interval Spaces. Nonlinear Anal. 2010, 72, 469-477. [CrossRef]

11. Rådström, H. An Embedding Theorem for Spaces of Convex Sets. Proc. Am. Math. Soc. 1952, 3, 165-169. [CrossRef]

(C) 2019 by the authors. Licensee MDPI, Basel, Switzerland. This article is an open access article distributed under the terms and conditions of the Creative Commons Attribution (CC BY) license (http:/ / creativecommons.org/licenses/by/4.0/). 